Running Head: BRIDGING PATTERNS

Bridging Patterns of Neurocognitive Aging Across the Older Adult Lifespan

Jenna L. Merenstein \& Ilana J. Bennett

Department of Psychology, University of California, Riverside

Address correspondence to:

Jenna L. Merenstein

Department of Psychology

University of California, Riverside

900 University Avenue

Riverside CA, 92521-0426

PH: 951-961-7181

Email: jklip001@ucr.edu

The authors declare no competing financial interests. 


\begin{abstract}
Magnetic resonance imaging (MRI) studies of brain and neurocognitive aging rarely include oldest-old adults (ages 80+). But predictions of neurocognitive aging theories derived from MRI findings in younger-old adults (ages $~ 55-80$ ) may not generalize into advanced age, particularly given the increased prevalence of cognitive impairment/dementia in the oldest-old. Here, we reviewed the MRI literature in oldest-old adults and interpreted findings within the context of regional variation, compensation, brain maintenance, and reserve theories. Structural MRI studies revealed regional variation in brain aging as larger age effects on medial temporal and posterior regions for oldest-old than younger-old adults. They also revealed that brain maintenance explained preserved cognitive functioning into the tenth decade of life. Very few functional MRI studies examined compensatory activity in oldest-old adults who perform as well as younger groups, although there was evidence that higher brain reserve in oldest-old adults may mediate effects of brain aging on cognition. Despite some continuity, different cognitive and neural profiles across the older adult lifespan should be addressed in modern neurocognitive aging theories.
\end{abstract}

Keywords (3-12): oldest-old, octogenarians, nonagenarians, neurocognitive aging, compensation, brain maintenance, brain reserve, anterior-to-posterior, advanced age, magnetic resonance imaging, neuroimaging 


\section{Bridging Patterns of Neurocognitive Aging Across the Older Adult Lifespan}

\section{Introduction}

Oldest-old adults beyond 80 years of age represent the fastest growing segment of the population in most developed countries (He and Muenchrath, 2011). However, previous neuroimaging studies have primarily related measures of brain aging to cognitive performance in younger-old adults aged $\sim 55-80$ years. Numerous impactful theories of neurocognitive aging have been derived from these earlier studies, but their predictions based on younger-old cohorts may not generalize to oldest-old cohorts. The few studies extending into advanced age primarily focus on disease-related brain and neurocognitive changes seen in oldest-old adults with cognitive impairment (Corrada et al., 2010, 2008; Yang et al., 2013). Even less is known about cognitively normal oldest-old adults, $\sim 50 \%$ of whom have no evidence of brain pathologies (Kawas et al., 2015). Furthermore, the large heterogeneity of normal brain aging across the older adult lifespan (Eavani et al., 2018; Poulakis et al., 2021) may differentially affect cognitive and neural measures in oldest-old compared to younger-old adults. It is therefore important to consider the degree to which extant neurocognitive aging theories account for findings reported in advanced age.

Magnetic resonance imaging (MRI) is a neuroimaging technique that is well suited for examining age-related differences in brain structure and function in advanced age and determining whether such neural differences are predictive of cognitive deficits (Hartel and Buckner, 2006; Young et al., 2020). Advantages of MRI include it being readily available, costeffective, and non-invasive relative to other neuroimaging techniques, such as positron emission tomography (PET), computerized tomography (CT), near infrared spectroscopy (NIRS), and electro- and magneto-encephalography (EEG/MEG). Individual MRI scans are relatively short 
(3-8 minutes), with structural scans requiring nothing more than having participants lie still for the duration of the scan session (15-45 minutes). Padding and other accommodations (e.g., blankets, nonferrous glasses, ear buds) can make the experience more comfortable (e.g., for those with spine curvature), not just more accessible, for individuals with various physical issues (e.g., vision problems, arthritis).

Multiple MRI modalities can also be acquired from participants during a single scanning session, which allows studies to obtain varied measures of brain structure and function. For example, one common MRI modality is high-resolution T1-weighted images, which can reveal age-related differences in the degree of atrophy (volume, morphometry) of gray and white matter (Anderson et al., 2005). Diffusion tensor imaging (DTI) provides more detailed estimations of microstructural tissue properties by measuring the jitter (diffusion) of molecular water (Beaulieu, 2002; Jones, 2008; Mori and Zhang, 2006). Damage to white matter tissue can be further probed by estimating the volume of white matter hyperintensities (WMH) using fluid attenuated inversion recovery (FLAIR) sequences (Lockhart and DeCarli, 2014). On the other hand, agerelated differences in brain activity can be inferred using functional MRI (fMRI), which provides estimates of the blood-oxygen-level-dependent (BOLD) signal that can either be acquired during performance of a cognitive task (i.e., task-based fMRI; Logothetis, 2008) or during rest (i.e., resting state fMRI; Cole et al., 2010). In addition to the advantages noted above, this array of modalities makes MRI an ideal tool to examine the neural mechanisms affected by advanced age and whether their contributions to cognition differ across the older adult lifespan.

Other reviews on the oldest-old have focused on broader neuroimaging findings (i.e., including both CT and PET studies; Woodworth et al., 2021), problems related to frailty and cardiovascular disease (Rosa et al., 2020), risk factors of dementia (Gardner et al., 2013; 
Paolacci et al., 2017; Pierce and Kawas, 2017), methodological considerations (Poon et al., 2007), and the epidemiology and pathology of dementia (Gardner et al., 2013; Kawas et al., 2021; Von Gunten et al., 2010; Yang et al., 2013). The current review will add to this literature by (1) reviewing neuroimaging studies of brain and neurocognitive aging in the oldest-old, (2) evaluating whether these findings align with select neurocognitive aging theories and findings in younger-old adults, and (3) providing methodological considerations and ideas for future neuroimaging research in the oldest-old. Ultimately, this review will demonstrate that the extant literature is sufficient large to identify areas of convergence and call for areas that will benefit from further study, with particular attention to the methodological concerns discussed here.

\section{Scope of Review}

We conducted our review between May and July 2021 with PubMed searches using both an age (“oldest old”, “old old", “very old”, “centenarians”, “nonagenarians”, “octogenarians”, “ag*ing”, "80 and over”) and MRI (“magnetic resonance imaging”, "MRI”, "brain”) term, with these searches repeated after adding a cognition term (“dementia", "cognition", "cognitive performance”). We selected studies that met the following criteria: (1) English language publications up to 2021, (2) involved human subjects, (3) original research reports, (4) included adults over 80 years old, and (5) examined effects of age on an MRI measure of brain structure or function and/or relationships between chronological age and cognitive performance. Studies were further limited to those assessing these effects within older age groups (e.g., ages 55+ years old), with lifespan studies (e.g., ages 20-80+ years) only included when their results were disaggregated by an oldest-old adult subgroup (e.g., conducting analyses with and without oldest-old adults or examining effects separately within oldest-old adults).

\subsection{Defining the "oldest-old"}


The definition of oldest-old adults varies across research groups, with the Sydney Memory and Aging Study (Piguet et al., 2003; Z. Yang et al., 2016b) and Health, Aging, and Body Composition Study (Rosano et al., 2005a, 2005b; Simonsick et al., 2001) including individuals greater than 80 years old, whereas The 90+ Study (Kawas and Corrada, 2006) is limited to individuals greater than 90 years of age. Because normal aging (e.g., neurodegeneration, small vessel disease) and dementia-related (e.g., amyloid-beta plaques, neurofibrillary tangles; Braak and Braak, 1997) pathology are both less prevalent in octogenarians (80-89 years) than nonagenarians (90-99 years)(Kawas et al., 2015; Yang et al., 2013), one could argue that the latter group represents a more stringent definition of the oldestold. Nonetheless, to better integrate findings across these cohorts, the current review defined oldest-old adults as individuals beyond 80 years of age.

Studying the oldest-old provides an opportunity to assess how their increased prevalence of cognitive impairment no dementia (CIND) affects MRI measures of brain aging (Brookmeyer et al., 2017; Corrada et al., 2010, 2008). Relative to oldest-old adults with normal cognition, those with CIND are at increased risk of progressing to dementia (Peltz et al., 2011), which we assume reflects a relatively greater accumulation of dementia-related pathology contributing to their clinical expression of cognitive deficits. To disentangle these distinct but related constructs, however, we suggest that future MRI studies examining cognitive status subgroup differences in this age group simultaneously assess Alzheimer's disease risk factors (e.g., e4 allele combination on the apolipoprotein [APOE] gene) and pathology (e.g., amyloid-beta).

\subsection{Overview of neurocognitive aging theories}

The body of this review is divided into four sections covering studies that assessed gray matter volumetry and morphometry (Section 3), white matter hyperintensities and microstructure 
(Section 4), fMRI activity (Section 5), or other MRI modalities (Section 6) in the oldest-old. Each section separately reviews the literature examining brain aging (Table 1), neurocognitive aging (Table 2), and cognitive status subgroups (Table 3 ) in the oldest-old using the corresponding MRI modality. Findings within each subsection are then discussed in relation to the following four theories, the latter of which is only discussed in the fMRI section (Table 4): regional variation of brain aging (Raz et al., 2010; West, 1996), brain maintenance (Nyberg et al., 2012), brain reserve (Barulli and Stern, 2013), and compensation (Cabeza, 2002; Cabeza et al., 2018; Grady, 2008; Reuter-Lorenz and Cappell, 2008). These neurocognitive aging theories were selected for their ability to make specific predictions that could be applied to MRI data in the oldest-old. Each theory is briefly introduced below.

\subsubsection{Regional variation of brain aging}

Numerous theories in younger-old adults propose that the magnitude of age effects varies across the brain, particularly in frontal and medial temporal regions. One influential theory, the frontal lobe hypothesis, proposed that healthy aging disproportionately affects anterior brain regions, resulting in worse performance on cognitive processes supported by the prefrontal cortex (e.g., executive functioning; West, 1996). An extension of this view proposes an anteriorto-posterior gradient in normal brain aging such that age effects in younger-old adults are largest and appear earliest in frontal regions, with parietal and occipital regions being relatively preserved until advanced age (Cabeza and Dennis, 2014; Davis et al., 2009; Head et al., 2004; Hedden and Gabrieli, 2004; Madden et al., 2009; Pfefferbaum et al., 2005). However, others report similarly large age-related differences in medial temporal regions such as the hippocampus and entorhinal cortex in younger-old adults, which have been associated with episodic memory deficits (for reviews, see Craik and Rose, 2012; Jagust, 2013; Tromp et al., 
2015).

An important question, particularly when applying these theories to the oldest-old, is whether frontal lobe atrophy occurs in both normal aging and dementia, whereas medial temporal lobe atrophy primarily occurs in individuals with dementia (Head et al., 2005; Hedden and Gabrieli, 2005; Resnick et al., 2003). This distinction arose from the latter regions being among the first to accumulate dementia-related pathologies (e.g., amyloid-beta plaques, neurofibrillary tangles; Braak and Braak, 1997). Yet other work suggests that frontal and medial temporal regions are similarly affected by normal aging (Fjell et al., 2014; Raz et al., 2010, 2005), as both regions have smaller diameter axons and lower oligodendrocyte-to-axon ratios making them more vulnerable to degeneration (Stebbins and Murphy, 2009), as well as a delayed time course for myelination (Bartzokis, 2004). The MRI literature in oldest-old adults may contribute to this debate by comparing medial temporal regions in individuals at low versus high risk for dementia, the latter of which includes those diagnosed with CIND.

Regional variation in brain aging within oldest-old adults may present as frontal and medial temporal vulnerability similar to younger-old adults, consistent with the notion that these regions decline across the older adult lifespan. Relative to younger-old adults, larger age effects in medial temporal regions may reflect an accumulation of dementia-related pathology in both cognitively normal and CIND oldest-old adults. However, an open question is whether additional regions that are relatively preserved in younger-old adults, such as parietal and primary sensory areas, are vulnerable in advanced age.

\subsubsection{Brain maintenance}

Brain maintenance is a theory proposing that younger-old adults with cognitive abilities similar to younger adults (e.g., 20-30 years) or better than age-expected norms experience 
minimal age-related brain changes and a relative lack of brain pathology (Nyberg et al., 2012; Nyberg and Pudas, 2018). In other words, these cognitively normal older adults have "maintained" a young-like brain, which is comparable to the term "resistance" often used in Alzheimer's disease biomarker research (Montine et al., 2019). For example, one study found similar prefrontal recruitment when comparing younger-old adults who performed well on a memory task to younger and middle-aged (e.g., 30-55 years) adults (Vidal-Piñeiro et al., 2018). These same top-performing younger-old adults also had no significant decline in memory performance and slower rates of entorhinal cortical atrophy measured over eight years, suggesting that these substrates helped preserve memory function. However, very few studies have interpreted similar effects in light of brain maintenance in advanced age.

Brain maintenance may generalize to oldest-old adults. Support for this view would include structural MRI studies finding that oldest-old adults with the best cognitive performance also have the largest brain volumes, intact tissue microstructure, and fewer WMH, especially longitudinally. It might also include fMRI studies finding that top-performing oldest-old adults with little structural degradation recruit similar brain networks to a comparable degree as younger age groups, as noted above.

\subsubsection{Brain reserve}

Brain reserve has been proposed as a mechanism to account for individual differences in cognitive aging in younger-old adults and is similar to the term "resilience" used in Alzheimer's disease biomarker research (Montine et al., 2019). The idea is that cognitive impairment will not be observable until changes in the brain, like those associated with aging and dementia, exceed some threshold that varies across individuals depending on their "brain reserve", such as their brain size, neurite density, or synaptic connections (Barulli and Stern, 2013). Thus, older 
individuals with high brain reserve may be cognitively normal despite having a large amount of age-related degradation or accumulation of pathology. This differs from brain maintenance, which proposes that individuals with normal cognition should have an absence of age-related brain changes or disease-related pathologies.

In the oldest-old, brain reserve theories may explain structural MRI studies finding that a given level of neural degradation (e.g., atrophy, WMH burden) results in cognitive impairment for individuals with low brain reserve, whereas those with high brain reserve will continue to present as cognitively normal (Barulli and Stern, 2013; Tucker and Stern, 2011). It may also be used to interpret fMRI studies finding that oldest-old adults with similar cognitive performance relative to younger age groups have similar or even reduced BOLD activity in the face of marked structural degradation. This pattern has previously been interpreted as a form of functional reserve against age-related brain changes, potentially reflecting more efficient use of the spared brain tissue in pre-existing networks (Barulli and Stern, 2013; Stern, 2006).

\subsubsection{Compensation}

As defined in a recent consensus paper (Cabeza et al., 2018), compensation theories propose that younger-old adults may compensate for the negative effects of brain aging by increasing activity in the same and/or additional brain regions relative to younger adults, allowing them to perform well on the cognitive tasks (Cabeza et al., 2018; Davis et al., 2008; Grady, 2008). One of the earliest compensation theories is Hemispheric Asymmetry Reduction in Older Adults (HAROLD; Cabeza, 2002), which described the finding that high performing older adults recruited bilateral prefrontal regions relative to younger adults and low performing older adults who recruited unilateral regions during memory performance (Cabeza et al., 1997). Compensation-Related Utilization of Neural Circuits Hypothesis (CRUNCH; Reuter-Lorenz and 
Cappell, 2008) further proposes that older adults show compensatory activity when task demands are low and they can perform well, but fail to show compensatory activity when task demands are high and they perform worse than younger adults. This is thought to result from older adults reaching a ceiling of neural resources that can be recruited when tasks are more difficult. As with regional variation theories, compensatory activity is often seen in frontal brain regions.

Compensatory neural activity in the oldest-old should look similar to patterns seen in younger-old adults, with more BOLD activity in individuals whose cognitive performance is comparable to younger age groups. However, it is possible that compensatory activity may not be seen in oldest-old adults if their performance is always worse than younger age groups. Such a finding would be consistent with $\mathrm{CRUNCH}$ if it reflects task demands being higher in advanced age.

\section{Gray matter volumetry and morphometry}

\subsection{Brain aging in the oldest-old}

Because the literature on white matter volume in the oldest-old consists of too few studies to draw consistent conclusions (Salat et al., 1999; Stickel et al., 2018; Z. Yang et al., 2016a), this section instead focused on studies assessing the effect of advanced age on gray matter volume and morphometry (cortical thickness, sulcal width). These studies have revealed three key findings: (1) negative effects of age on volume were consistently seen for the hippocampus, (2) less consistent and possibly weaker age effects on volume and mophometry were seen in other medial temporal and frontal regions, and (3) the negative effects of age were comparable in oldest-old adults with normal and superior cognitive status. The literature supporting these findings is described below and summarized in Table 1.

Most studies reported that advanced age was accompanied by smaller volume of the 
hippocampus (Mueller et al., 1998; van Bergen et al., 2018; Z. Yang et al., 2016a), a medial temporal structure commonly linked to memory ability, with this negative effect of age on hippocampal volume being significantly greater than in the prefrontal cortex (Yang et al., 2016a). Together, this suggests that there are consistent and large effects of advanced age on hippocampal volume relative to younger-old adults, similar to lifespan studies extending into advanced age (Jernigan et al., 2001; Langnes et al., 2020).

As in the hippocampus, advanced age-related degradation has been observed in other medial temporal and brain-wide structures. Relative to younger-old adults, oldest-old adults have significantly smaller volumes (Brickman et al., 2008) and greater thinning (Z. Yang et al., 2016a) of the entorhinal cortex, as well as smaller temporal lobe volumes (Mueller et al., 1998). There are also longitudinal decreases in temporal (and frontal) cortical thickness when following oldest-old adults over a four-year period (Li et al., 2020). Although at least one study found no significant effect of age on entorhinal volume within older adults (van Bergen et al., 2018). Beyond medial temporal structures, the sulcal width of both anterior (e.g., anterior cingulate, superior frontal) and posterior (e.g., intraparietal, posterior cingulate) regions was greater for oldest-old than younger-old adults (Tang et al., 2021). Indeed, effects of advanced normal aging on anterior neural tissue appears to be distinct from disease, as one study observed significantly greater volume loss in the prefrontal cortex for cognitively normal oldest-old adults than younger-old adults with normal cognition or those with Alzheimer's disease (Salat et al., 1999). Together, these findings suggest brain-wide effects of advanced age on gray matter volume and morphometry.

When examining these age effects across cognitive status subgroups, one study observed that even older adults with superior cognitive status experienced whole brain volume loss and 
cortical thinning, with the largest age effects in the hippocampus and entorhinal cortex (Z. Yang et al., 2016a). This finding suggests that some degree of advanced age-related degeneration in the hippocampus, among other brain regions, may be characteristic of normal aging. However, additional research with oldest-old adults across cognitive status subgroups is needed to better understand the extent to which volume and morphometry differences reflect normal aging or preclinical dementia, especially those that can also assess Alzheimer's disease risk factors and pathology (e.g., APOE genotype, amyloid-beta).

In summary, the current findings suggest a more brain-wide vulnerability in advanced age that is especially marked in the hippocampus. This pattern of results is not inconsistent with neurocognitive aging theories in younger-old adults, such as the anterior-to-posterior gradient (Davis et al., 2009; Head et al., 2004; Hedden and Gabrieli, 2004; Madden et al., 2009), in that advanced age effects on volume and morphometry also extended to anterior and posterior cortical regions (Li et al., 2020; Tang et al., 2021; Z. Yang et al., 2016a). Yet this view does not account for the predominant pattern of hippocampal degradation seen in advanced aging (Z. Yang et al., 2016a). Instead, the findings reviewed here suggest that an anterior-to-posterior gradient may be more common in early aging. Future studies examining age effects using the same structural imaging modality across the older adult lifespan are needed to better understand the time course of degradation in anterior (frontal cortex), medial temporal (hippocampus), and posterior (parietal cortex) regions. In turn, extant theories of regional variation in brain aging may need to account for the increased hippocampal-specific and brain-wide susceptibility to advanced age.

\subsection{Neurocognitive aging in the oldest-old}

Studies examining the effect of brain volume and morphometry on cognition in advanced 
age have primarily focused on episodic memory and processing speed performance. Their results have predominantly revealed positive associations with hippocampal volume or medial temporal cortical thickness using a variety of study designs (cross-sectional, longitudinal) and age groups (entire lifespan, older adult lifespan, oldest-old only). The literature supporting these findings is described below and summarized in Table 2.

Slower rates of episodic memory decline assessed longitudinally have been reported within oldest-old adults with fewer changes in hippocampal volume (Legdeur et al., 2019) and higher baseline medial temporal and anterior cingulate cortical thickness (Pelkmans et al., 2021). Slower rates of memory decline have also been associated with larger baseline whole brain volume across the older adult lifespan (Carmichael et al., 2012). Cross-sectional studies similarly report that oldest-old adults with larger hippocampus volumes have better episodic memory performance (Eguchi et al., 2019) and faster processing speeds (Legdeur et al., 2020; Pelkmans et al., 2021), comparable to what has been reported in younger-old adults (Carr et al., 2017; Gorbach et al., 2017; O'Shea et al., 2016). Larger volume of the hippocampus was also found to predict better memory performance in individuals across the lifespan (ages 4-93 years; Langnes et al., 2020). However, the effects in anterior hippocampus were driven by individuals aged $80+$ years, suggesting that this subregion of the hippocampus may be especially important for memory function in advanced age.

In summary, larger gray matter volumes and morphometry, primarily of the hippocampus and medial temporal lobe, relate to better performance on episodic memory and processing speed tasks across the lifespan and into advanced age. Because these studies revealed better cognitive performance in oldest-old adults with either minimal age-related brain changes assessed longitudinally or minimal structural degradation assessed cross-sectionally (e.g., larger volume, 
thicker cortex), their findings are consistent with the brain maintenance theory (Nyberg et al., 2012). Specifically, as in younger-old adults, maintenance of a young-like brain in advanced age may prevent cognitive aging. Future work extending beyond medial temporal regions and episodic memory processes can help test the functional role of frontal and posterior brain aging (e.g., using executive function or attention-based paradigms) in oldest-old relative to younger-old adults, especially those using longitudinal designs.

\subsection{Cognitive status subgroups in the oldest-old}

Several studies have compared volumetry and morphometry measures between subgroups of oldest-old adults that differ in cognitive status, defined as normal cognition, cognitive impairment, or dementia. These studies have revealed similar effects of cognitive status across the older adult lifespan in medial temporal regions (hippocampus, entorhinal cortex, parahippocampal gyrus), although the effect of cognitive status on volume and morphometry in cortex (temporal, parietal) may be smaller in oldest-old than younger-old adults. The literature supporting these findings is described below and summarized in Table 3.

Larger hippocampal volumes are consistently seen for oldest-old adults with normal cognition relative to those with cognitive impairment or dementia (Barkhof et al., 2007; Gosche et al., 2002; Holland et al., 2012; Lopez et al., 2014; Z. Yang et al., 2016b), similar to findings in younger-old adults (Apostolova et al., 2012; Z. Yang et al., 2016b). Larger volumes of the hippocampus, as well as entorhinal cortex and parahippocampus gyrus, have also been seen for oldest-old adults with preserved general cognitive performance over 10 years than those who declined, with comparable effects in younger-old adults (Rosano et al., 2012). However, at least one study found that brain regions showing a volumetric effect for normal versus impaired cognitive status differed in oldest-old (hippocampus, inferior frontal gyrus, temporal pole) 
relative to younger-old (putamen, parahippocampal gyrus, cortex) adults (Z. Yang et al., 2016b). Smaller differences between cognitively normal and dementia subgroups have also been reported for thickness (Stricker et al., 2011) and volume (Holland et al., 2012) of temporal-parietal regions for oldest-old relative to younger-old adults. A more complete picture of the structural signatures of normal aging versus dementia will require future studies that compare cognitive status subgroups in younger-old and oldest-old populations using the same MRI metrics.

In summary, these findings are generally in line with brain reserve, which would expect cognitively normal individuals to have higher brain reserve (e.g., larger brain volumes) than those with cognitive impairment or dementia. Whereas some degree of atrophy may be characteristic of normal aging (Z. Yang et al., 2016a), more accelerated degradation as a function of cognitive status, particularly in the medial temporal lobe, is consistent with its role in dementia across the older adult lifespan. However, these findings further suggest that levels of reserve are not uniform across the brain and that regional differences may be exacerbated in advanced age, which warrants further investigation.

\section{White matter hyperintensities and microstructure}

\subsection{Brain aging in the oldest-old}

Effects of advanced age on WMH and microstructure have been assessed across the older adult lifespan and within the oldest-old only, using a variety of cognitive status subgroups (superior, normal, impaired). These studies have revealed three key findings: (1) WMH accumulate more in oldest-old relative to younger-old adults predominantly in posterior brain regions, (2) worse tissue microstructure (i.e., lower fractional anisotropy, FA; higher diffusivity) is seen in oldest-old relative to younger-old adults that is most prominent in medial temporal regions, and (3) these effects of age on $\mathrm{WMH}$ and microstructure do not vary between oldest-old 
adults with normal or impaired cognitive status. The literature supporting these findings is described below and summarized in Table 1.

One study examining WMH across the older adult lifespan found quadratic age-related differences for all four cortical lobes (Z. Yang et al., 2016a), indicating an accelerated accumulation of WMH in advanced age. Specific vulnerability of the frontal lobe was supported by one study limited to this region finding significantly higher WMH burden for oldest-old adults $>91$ years than those $<90$ years old (Polvikoski et al., 2010). However, when examining WMH across the brain within oldest-old adults, there was significantly higher WMH burden in the parietal than the frontal lobe (Piguet et al., 2003). Thus, whereas there are larger brain-wide WMH differences in advanced age relative to younger adults, parietal regions may be most sensitive to age-related WMH accumulation in the ninth and tenth decades.

Studies examining white matter microstructure have reported negative age effects on frontal, temporal, and parietal regions across the older adult lifespan (Lövdén et al., 2013), as well as within oldest-old adults over a two-year period (Lövdén et al., 2014). Our own work in younger-old and oldest-old adults revealed quadratic age-related differences in white matter microstructure across the brain that were more pronounced in advanced age, with the largest effects seen for the medial temporal lobe (Merenstein et al., 2021). These findings extended earlier work in which we found the largest age effects for medial temporal (fornix) and posterior (splenium) white matter tracts within oldest-old adults (Bennett et al., 2017). As such, there are consistently large effects of advanced age on medial temporal white matter relative to youngerold adults and even amongst only oldest-old adults.

There is some evidence that advanced age-related WMH accumulation and microstructural degeneration is not driven by oldest-old with cognitive impairment or dementia. 
For example, the previously mentioned age-related increases in brain-wide WMH were observed even in older adults with superior cognition (Z. Yang et al., 2016a), and the larger age effect on parietal than frontal WMH was independent of APOE genotype (Piguet et al., 2003). Moreover, our findings of brain-wide age effects on microstructure across the older adult lifespan (Merenstein et al., 2021) and within only oldest-old adults (Bennett et al., 2017) did not change after excluding oldest-old adults diagnosed with CIND. Thus, brain-wide microstructural degradation seen in advanced age cannot solely be attributed to cognitive dysfunction or increased dementia risk in this age group.

In summary, finding greater age effects on posterior regions differs from the predominantly frontal findings in younger-old adults, but together they support an anterior-toposterior gradient to white matter aging (Head et al., 2004; Hedden and Gabrieli, 2004; Madden et al., 2009). Importantly, there was nonlinear white matter degradation in medial temporal and posterior regions within oldest-old adults regardless of CIND or superior cognitive status, which might reflect an acceleration of normal aging processes in advanced age (e.g., demyelination, myelin ballooning, cardiovascular damage; Peters, 2002; Wardlaw et al., 2015). These findings are consistent with the gray matter volumetry and morphometry studies reviewed in Section 3.1, thereby providing converging evidence for a brain-wide vulnerability in advanced age that spans multiple neural substrates captured by these different MRI modalities. Because most studies examining WMH and microstructure in the oldest-old have used cross-sectional designs (c.f., Lövdén et al., 2014), this line of work could be progressed by future longitudinal studies that can track these brain aging effects over time into advanced age.

\subsection{Neurocognitive aging in the oldest-old}

Numerous cross-sectional and longitudinal studies have shown that fewer WMH and 
better microstructure (e.g., higher FA, lower diffusivity) relates to better cognitive performance into advanced age, with the most studied cognitive domains being episodic memory, executive functions, and processing speed. The literature supporting these findings is described below and summarized in Table 2.

For episodic memory, oldest-old adults with slower rates of memory decline had fewer baseline WMH across the brain (Pelkmans et al., 2021), with this association being particularly strong within the frontal lobe (Piguet et al., 2003). Better episodic memory performance has also been related specifically to better medial temporal white matter microstructure (Merenstein et al., 2021), as well as better hippocampal gray matter microstructure (Reas et al., 2021), across the older adult lifespan. For executive functions, older adults who accumulated fewer brain-wide WMH over time had smaller declines in performance (Carmichael et al., 2012), although the association between WMH burden and executive functions may be weaker in oldest-old adults (Legdeur et al., 2019). For processing speed, faster performance within oldest-old adults has been associated with fewer brain-wide WMH (Pelkmans et al., 2021) and better microstructure of the corticospinal tract (Lövdén et al., 2014), uncinate and superior longitudinal fasciculi (Rosano et al., 2015), and whole brain white matter (Venkatraman et al., 2011). Associations between microstructure and processing speed in individuals across the older adult lifespan remained significant after accounting for the future development of dementia (Haynes et al., 2017) or excluding individuals meeting criteria for preclinical dementia (Laukka et al., 2013), suggesting that these effects are not solely driven by early cognitive impairment.

Altogether, these patterns would be predicted by brain maintenance theory, where preserved cognitive functioning should be observed among older adults with minimal changes in markers of brain aging. One interesting finding that requires further investigation is reports of 
race (Liu et al., 2015) and sex (Reas et al., 2021) differences in relationships between performance and WMH or microstructure. Findings such as smaller WMH volumes relating to faster processing speeds for Black but not White younger-old and oldest-old adults (Liu et al., 2015) may be due to the larger WMH volumes seen among Black (and Hispanic) relative to White individuals across the older lifespan (Brickman et al., 2008). Future studies using more diverse oldest-old samples are needed to determine whether race and sex moderate the association between the previously reported MRI markers and cognition in advanced age.

\subsection{Cognitive status subgroups in the oldest-old}

Studies comparing WMH and microstructure measures between subgroups of oldest-old adults that differ in cognitive status (normal cognition, cognitive impairment, dementia) report a weakened ability of these measures to differentiate these subgroups in the oldest-old. The literature supporting this finding is described below and summarized in Table 3.

A handful of studies have shown that whole brain WMH volumes do not significantly differ between cognitively normal and impaired subgroups within oldest-old adults (Tanskanen et al., 2013; Zamboni et al., 2019) or across younger-old and oldest-old adults (Z. Yang et al., 2016b). Longitudinally, baseline whole brain WMH burden in individuals across the older adult lifespan was comparable for those who had preserved and declining general cognitive performance over a 10-year period (Rosano et al., 2012). Comparable effects have been reported for whole brain white matter microstructure, which differed between cognitively normal and impaired subgroups of younger-old, but not oldest-old, adults (Zamboni et al., 2019). However, region-specific differences between oldest-old adults with normal and impaired cognition may have been obscured by the use of whole brain WMH measures as one study limited to the frontal lobe observed fewer WMH for oldest-old adults with normal cognition than those diagnosed 
with Alzheimer's disease (Polvikoski et al., 2010).

Thus, although individual differences in $\mathrm{WMH}$ or microstructure significantly relate to cognitive performance in both cognitively normal and impaired oldest-old adults (see Section 4.2), these white matter metrics do not differentiate cognitive status subgroups in advanced age. This pattern may indicate that the contribution of white matter to cognitive dysfunction in clinically impaired oldest-old adults is somewhat modest or that the cognitive status subgroups are defined by additional factors not captured by the measures of cognitive performance (e.g., impaired activities of daily living). Regardless, finding comparable WMH accumulation (De Leeuw et al., 2001; Kawas et al., 2015) and microstructural degradation (Bennett et al., 2017; Merenstein et al., 2021) in oldest-old adults with normal and impaired cognition may reflect higher brain reserve in the former group as they show preserved cognition in spite of white matter degradation. This interpretation would benefit from future work testing whether neural substrates beyond WMH and microstructure (e.g., functional activity and connectivity, larger brain volumes, low levels of amyloid-beta plaques) capture similar patterns of brain reserve that differ between these cognitive status subgroups in advanced age.

\section{Functional MRI studies}

\subsection{Brain and neurocognitive aging in the oldest-old}

Beyond the large number of structural studies whose samples extend into advanced age, there are also eight fMRI studies that have been conducted in oldest-old adults. Most task-related fMRI studies report decreased BOLD activity into advanced age that is independent of preserved or impaired performance, whereas resting-state fMRI studies find a mixture of age-related decreases (default mode) and increases (frontoparietal, motor) in functional connectivity. The literature supporting these findings is described below and summarized in Table 4. 
Two studies compared BOLD activity between younger-old and oldest-old adults during recognition memory performance, with both controlling for age-related differences in whole brain volume (Beeri et al., 2011; Wang et al., 2009). When oldest-old adults had poorer memory performance than younger-old adults, it was accompanied by less activity in hippocampal, temporal, and parietal regions, but comparable activity in frontal cortex (Beeri et al., 2011). When matching younger-old and oldest-old adults on memory performance, results similarly revealed age-related decreases in medial parietal activity but comparable activity in frontal and lateral parietal regions (Wang et al., 2009).

Other studies have examined BOLD activity across age groups as a function of task demands. One study compared oldest-old to younger adults who performed an executive control task, in which participants executed a motor response to a target stimulus that was preceded by a congruent (low load) or incongruent (high load) cue (Rosano et al., 2005a). Across load conditions, the oldest-old had similarly high accuracy levels as younger adults, but lower activity in the dorsolateral prefrontal and posterior parietal cortices. However, both age groups showed increased recruitment of these same regions for the high versus low load condition, and oldestold adults with the greatest load-related parietal activity had the best performance. Another study compared adults across the lifespan (ages 20-89 years) during performance of a semantic judgment task, where participants made "living" versus "nonliving" judgments to words that were more (high load) or less (low load) ambiguous (Kennedy et al., 2015). Results also revealed similarly high accuracy levels across age groups, but performance was instead accompanied by decreased recruitment of frontal, temporal, and parietal regions at higher versus lower task loads, especially within the oldest-old subgroup (ages $80+$ years).

Whereas the aforementioned fMRI studies focused on BOLD activity during performance 
of a task, at least two studies examined patterns of synchronous activity between regions while participants rested (i.e., functional connectivity). Across the older adult lifespan, age-related differences were seen in connectivity of the default mode network, such that older adults had lower connectivity between regions such as the medial prefrontal cortex, posterior cingulate, and precuneus (Jiang et al., 2020; Li et al., 2020). The cross-sectional study also found greater bilateral frontoparietal connectivity that predicted better visuospatial performance for the oldestold (Jiang et al., 2020), whereas the longitudinal study observed age-related increases in connectivity between the insula and supplementary motor area, with precuneus connectivity relating to changes in general cognitive performance (Li et al., 2020). Results of both studies were independent of whole brain volume and comparable to findings within younger-old and oldest-old adults with Alzheimer's disease (e.g., in the default mode network; Prawiroharjo et al., 2020), suggesting that they are not driven by cognitive dysfunction.

In summary, the task-related findings differ from the age-related increases in BOLD activity typically seen in high-performing younger-old adults, as well as the relatively consistent increases in BOLD activity as a function of task demands in younger age groups, and therefore contrast with compensation theories (Cabeza et al., 2018; Grady, 2008). They also differ from brain maintenance, which would have instead predicted similar BOLD activity and comparable cognitive performance in oldest-old and younger groups. To some degree, these interpretations depend on the reference group, as differences in the oldest-old were more widespread when compared to younger adults (Kennedy et al., 2015; Rosano et al., 2005a) than younger-old adults (Beeri et al., 2011; Wang et al., 2009). Whereas compensatory fMRI activity is commonly attributed to an increase in neural activity in younger-old adults who perform as well as younger adults, some have proposed that compensatory activity follows an inverted U-shaped pattern 
across the entire adult lifespan (Cabeza and Dennis, 2014; Scheller et al., 2014). Such a pattern could be roughly approximated using both younger and younger-old comparison groups, which may reveal comparable neural activity in younger and high performing oldest-old adults, both of which differ from the increased activity seen in high performing younger-old adults. However, a fuller characterization of this U-shaped function will require a lifespan sample.

Given the relatively smaller fMRI literature, it remains somewhat unclear whether decreased BOLD activity reflects a maximization of neural resources in oldest-old adults (Reuter-Lorenz and Cappell, 2008) or whether they have higher brain reserve that protects against the effects of atrophy (Barulli and Stern, 2013). The latter would allow for efficient engagement of neural networks, which was supported by resting state studies finding stronger connectivity in the oldest-old (Jiang et al., 2020; Li et al., 2020). Ultimately, additional fMRI studies across the entire lifespan are needed to tease apart these possibilities, especially those using tasks with varied cognitive demands to prevent ceiling (younger adults) and floor (oldestold adults) effects or allow age groups to be matched on performance (Wang et al., 2009).

\subsection{Cognitive status subgroups in the oldest-old}

Only one study examined BOLD activity between cognitive status subgroups in advanced age, which is summarized in Table 4. During performance of the same executive control task described above (Rosano et al., 2005b), greater activity was seen in dorsolateral prefrontal and posterior parietal cortices for oldest-old adults with impaired versus normal cognition. Because there were no group differences in performance, it suggests that this response was compensatory. Thus, relative to cognitively normal younger-old and oldest-old adults, cognitively impaired oldest-old adults appear to show compensatory activity in parietal, not just frontal, regions.

\section{Other MRI modalities}


Beyond the select MRI modalities reviewed above, at least two studies examined the extent to which certain genotypes contributed to brain or neurocognitive aging in younger-old and oldest-old adults (Papenberg et al., 2015; Stickel et al., 2018). Two genes of particular interest are COMT (Catechol-O-Methyltransferase), which is implicated in neuromodulation of the prefrontal cortex and executive functions, and KIBRA, which is named for its role in producing proteins expressed in the kidneys and brain and has been implicated in episodic memory. Across studies, only oldest-old adults with the more favorable allele combination for COMT (Papenberg et al., 2015) or KIBRA (Strickel et al, 2018) had better prefrontal white matter microstructure or greater frontal and occipital volume, respectively. Having desirable allele combinations may therefore be a mechanism by which cognitively normal oldest-old adults maintain brain structure in advanced age.

Susceptibility-weighted imaging is another MRI modality of interest given its sensitivity to iron, which is known to accumulation with age and is thought to contribute to neurodegeneration via inflammation (Venkatesh et al., 2021; Zecca et al., 2004). Age-related increases in iron have been observed in oldest-old adults within the putamen (van Bergen et al., 2018), a subcortical structure known to gradually accumulate iron across the younger adult lifespan (Hallgren and Sourander, 1958). However, this same study reported significantly less iron accumulation for oldest-old than younger-old cognitively normal adults in frontal, parietal, and temporal cortices (van Bergen et al., 2018). Intriguingly, because cortical iron loads are relatively low up until midlife and then increase across older adulthood (Acosta-Cabronero et al., 2016; Zecca et al., 2004), this cannot explain why a sample limited to older adults would paradoxically find age-related decreases in iron for cognitively normal oldest-old adults (van Bergen et al., 2018). Instead, minimal cortical iron accumulation may be a marker of sustained 
cognitive functioning in the ninth and tenth decades of life, which has implications for brain maintenance theory.

\section{Methodological Considerations}

General methodological constraints when conducting research on the oldest-old have been reviewed elsewhere. For example, the oldest-old are often extremely frail, making it difficult for them to travel to and navigate university testing sites (Rosa et al., 2020). There is also a strong sampling bias for oldest-old adults that are female, Non-Hispanic Whites, and have high educational attainment (Gardner et al., 2013; Poon et al., 2007). Here, we will discuss five additional methodological considerations specific to conducting neuroimaging research in the oldest-old: feasibility, vascular disease, iron accumulation, the presence of multiple pathologies, and the choice of reference group.

\subsection{Feasibility}

A potential limitation to obtaining MRI data in the oldest-old is their heightened frailty and the need for participants to lay in the supine position for an extended period. Comfort can be maximized by using head padding, blankets, and leg cushions, and one study indeed reported similar overall comfort levels during MRI scanning (up to 1 hour) for younger-old and oldest-old participants (Wollman et al., 2004). However, the same study did find that the oldest-old were more bothered by MRI scanner noise, long scan times, and laying down. Researchers therefore need to weight the benefits of a shorter scan time (e.g., participant satisfaction, better data quality from less motion) with the cost of potentially reducing the number or resolution of scans. They might also wish to consider splitting higher-resolution acquisition sequences into multiple sessions, although this introduces the potential cost of losing data due to attrition. 
interested and able to participate in research, feasibility then shifts to decisions about which MRI modalities to acquire. Structural MRI scans place minimal demands on participants beyond those for safely being scanned (e.g., not home bound, no metal, ability to lay supine). In contrast, taskrelated fMRI scans may be hampered by cognitive and physical (e.g., arthritis, vision and/or hearing problems) conditions that makes it difficult to understand task instructions and respond to stimuli via MR-compatible button-box. Researchers might therefore consider using extensive practice trials before entering the scanner and increasing the response time windows when asking oldest-old adults to perform cognitive tasks in the scanner. Functional MRI data also requires longer scan times as there are several structural images that are also needed for preprocessing (e.g., registration, region of interest, tissue segmentation). Nonetheless, these types of studies are crucial for understanding brain aging in the oldest-old and could be made possible via largescale, open access datasets, such as the Lifespan Human Connectome Project Aging (Bookheimer et al., 2019).

An alternative neuroimaging approach to study brain activity in the oldest-old is functional near-infrared spectroscopy (fNIRS), which uses near-infrared light to assess hemodynamic activity. One previous study used a portable fNIRS device to study a communitybased sample of oldest-old adults at their personal residences, finding significant frontal activity during executive function performance that positively correlated with age (Huppert et al., 2017). This approach cannot fully replace fMRI, however, because it is limited to recording signals from cortical regions near the skull and cannot detect signals from deeper brain regions that are especially affected by advanced age, such as the hippocampus.

\subsection{Effects of vascular disease}

Another notable consideration when conducting MRI research on the oldest-old is the 
increased prevalence of vascular disease in advanced age, which accounts for some variance in cognitive decline (Rosa et al., 2020). Cardiovascular damage caused by small vessel disease and (micro)infarcts appears as hyperintensities on MR images (Wardlaw et al., 2015), thereby resulting in a decreased gray to white matter intensity ratio as age increases (Salat et al., 2009). This lack of differentiation between tissue types may lead to decreased precision of automated segmentation algorithms and registration pipelines used in MRI studies, affecting regional estimates for oldest-old relative to younger-old adults. Registration procedures based on white, rather than gray, matter boundaries may be more robust to pathologies and artifacts in MRI data of the oldest-old (Greve and Fischl, 2009).

Because the BOLD response indirectly measures neural activity as the ratio of oxygenated to deoxygenated blood, age-related vascular damage to the capillary beds feeding neural systems should also be considered when acquiring and interpreting fMRI data in the oldest-old (Kannurpatti et al., 2010; West et al., 2019). This vascular damage leads to a decreased ability to regulate neuronal homeostasis and energy demands, known as neurovascular coupling (Tarantini et al., 2017). Although the effects of age on neurovascular coupling do not appear to directly affect BOLD activity in younger-old adults (e.g., Grinband et al., 2017), this has not yet been tested in oldest-old adults. Future studies could estimate cardiovascular contributions to BOLD activity in advanced age (e.g., a breath holding task), although this will come at the expense of a slightly longer scanning protocol (Kannurpatti et al., 2010).

\subsection{Accumulation of iron}

Iron accumulation in the aging brain should also be considered as it may have a larger effect on the MR signal in oldest-old than younger-old adults. Specifically, the presence of iron can attenuate the MR signal at acquisition (Haacke et al., 2005) and influence measures of brain 
structure and function across regions that differentially accumulate iron across the lifespan (Hallgren and Sourander, 1958). Neuroimaging studies comparing younger-old and oldest-old adults may therefore be more accurate in regions that exhibit minimal (e.g., hippocampus) or gradual accumulation of moderate amounts of iron throughout the lifespan (e.g., caudate, putamen) compared to regions that accumulate large amounts of iron by early adulthood (e.g., globus pallidum). Importantly, quantitative susceptibility mapping sequences can be used to estimate iron burden so that the effect of iron on the MR signal can be statistically accounted for (Ruetten et al., 2019).

\subsection{Multiple pathologies}

Another consideration is that the oldest-old may represent a distinct pathophysiological population, leading to multiple or even different neurobiological substrates contributing to a given MRI measure. Specifically, numerous dementia-related pathologies (e.g., amyloid-beta plaques, neurofibrillary tangles) are common in advanced age, even in individuals with normal cognition (Kawas et al., 2015; Yang et al., 2013). Because the high prevalence of dementiarelated pathology in the oldest-old does not always manifest as observable cognitive impairment, the distinction between normal and disease-related brain aging is less clear compared to youngerold adults. To ensure generalizability of future studies to this entire population, special care should be taken to recruit well-characterized oldest-old adults both with and without cognitive impairment or dementia, as well as to include measures of Alzheimer's disease risk factors (e.g., APOE genotype) and pathology (e.g., amyloid-beta) when possible.

\subsection{Reference group}

Finally, an open question is whether the reference group for the oldest-old (80+) should be younger (20-30s) or younger-old ( $55-80)$ adults. Younger adults would be analogous to the 
reference group used in studies of younger-old adults and could therefore detect comparable age effects, but could miss age effects that are unique to oldest-old relative to younger-old adults. In contrast, because younger-old adults have already experienced some of the deleterious effects of brain aging, comparisons would only be sensitive to advanced age effects of sufficient magnitude, which may be underestimated. As such, future MRI studies disaggregating their results across each of these age groups will be fundamental to obtaining a more holistic view of advanced brain and cognitive aging (e.g., testing the proposed U-shaped function of compensatory activity in relation to age; Cabeza and Dennis, 2014).

\section{Future Directions}

\subsection{Beyond brain macrostructure}

Prior MRI studies in the oldest-old have largely focused on volumetry, WMH, and traditional DTI-derived measures of microstructure, which may be due in part to the ease of acquiring these data using clinical scanners. Going forward, theories of neurocognitive aging will benefit from studies assessing the effect of advanced age on other neural substrates. For example, future work using more advanced multi-shell diffusion imaging data acquisition and analyses (e.g., neurite orientation, density, and dispersion index, NODDI; Zhang et al., 2012) may better capture the multifaceted effects of brain aging in the oldest-old (Reas et al., 2021). Future studies might also consider prioritizing the collection of task-related fMRI data in this age group, as less is known about the functional substrates supporting cognitive performance in advanced age.

\subsection{Multimodal imaging}

Extant neurocognitive aging theories would also benefit from studies assessing interactions among multiple neuroimaging markers in advanced age as the majority of the literature reviewed here focused on a single imaging modality. One multimodal study found 
hippocampus atrophy, brain-wide $\mathrm{WMH}$, and beta-amyloid accumulation in oldest-old adults, but these measures were not correlated with each other (Lopez et al., 2014). Further evidence that they may be sensitive to different neural substrates is that each MRI measure was uniquely associated with cognitive status (Lopez et al., 2014) and individual differences in cognitive performance (Legdeur et al., 2020) in the oldest-old. Yet this interpretation would benefit from replication across age groups and brain regions. Other multi-modal approaches could examine relationships between brain structure and function in advanced age. To this end, the fMRI studies reviewed above were able to demonstrate that patterns of neural activity were independent of whole brain volume (Beeri et al., 2011; Jiang et al., 2020; Li et al., 2020; Wang et al., 2009). Future work combining DTI and fMRI could test whether decreased BOLD activity in oldest-old adults is instead driven by microstructural degradation of the white matter pathways connecting gray matter regions (Salat, 2011).

\subsection{Neuroimaging-neuropathology associations}

Given their advanced age, the oldest-old present a unique opportunity to acquire in vivo neuroimaging data and ex vivo pathological data in the same individuals. Several efforts are already underway to collect these data in advanced age, including The 90+ Study (Kawas and Corrada, 2006). Such datasets will be invaluable for validating neuroimaging markers (e.g., tissue volume, WMH, iron content, myelin content) against underlying neural substrates (e.g., loss or shrinkage of neurons or their processes, demyelination, perivascular space expansion, white matter disease, vascular damage). These data will also be vital for differentiating normal from pathological brain aging, such as whether the effects of advanced age on medial temporal and posterior brain structure are driven by different amounts or types of pathologies in individuals across cognitive status subgroups. 


\section{Conclusion}

Determining whether advanced age differentially affects MRI markers of brain and neurocognitive aging is crucial because previous neuroimaging research in younger-old adults may not generalize to individuals across the older adult lifespan. The current review supports the notion that there is regional variation in the magnitude of brain aging, such that frontal regions remain vulnerable across the older adult lifespan, whereas medial temporal and posterior regions become more vulnerable in the oldest-old. This review also found little support for compensation theories in the oldest-old, which differs from the large literature in younger-old adults, although this conclusion was based on very few fMRI studies. Nonetheless, our review did reveal strong support for the notion that both brain maintenance and brain reserve can explain sustained cognitive functioning in advanced age. In summary, older adults who reach the eighth through tenth decades of life exhibit different cognitive and neural profiles than the 60- or 70-year-olds represented in most previous MRI aging research (Figure 1) and these advanced age-related differences should be reflected in re-evaluations of current neurocognitive theories of aging. 
Acknowledgements: The authors thank Seerat Kang, Joanne Ha, and Aditi Bhat for their assistance with the literature review. This work was supported by F31 AG071189 (Merenstein). 


\section{References}

Acosta-Cabronero, J., Betts, M.J., Cardenas-Blanco, A., Yang, S., Nestor, P.J., 2016. In Vivo MRI Mapping of Brain Iron Deposition across the Adult Lifespan. J. Neurosci. 36, 364374. https://doi.org/10.1523/JNEUROSCI.1907-15.2016

Anderson, V.C., Litvack, Z.N., Kaye, J.A., 2005. Magnetic resonance approaches to brain aging and Alzheimer disease-associated neuropathology. Top. Magn. Reson. Imaging 16, 439452. https://doi.org/10.1097/01.RMR.0000245458.05654.D0

Apostolova, L.G., Green, A.E., Babakchanian, S., Hwang, K.S., Chou, Y.-Y., Toga, A.W., Thompson, P.M., 2012. Hippocampal Atrophy and Ventricular Enlargement in Normal Aging, Mild Cognitive Impairment (MCI), and Alzheimer Disease. Alzheimer Dis. Assoc. Disord. 26, 17-27. https://doi.org/10.1097/WAD.0b013e3182163b62

Barkhof, F., Polvikoski, T.M., Van Straaten, E.C.W., Kalaria, R.N., Sulkava, R., Aronen, H.J., Niinistö, L., Rastas, S., Oinas, M., Scheltens, P., Erkinjuntti, T., 2007. The significance of medial temporal lobe atrophy: A postmortem MRI study in the very old. Neurology 69, 1521-1527. https://doi.org/10.1212/01.wnl.0000277459.83543.99

Bartzokis, G., 2004. Age-related myelin breakdown: a developmental model of cognitive decline and Alzheimer's disease. Neurobiol. Aging 25, 5-18. https://doi.org/10.1016/J.NEUROBIOLAGING.2003.03.001

Barulli, D., Stern, Y., 2013. Efficiency, capacity, compensation, maintenance, plasticity: Emerging concepts in cognitive reserve. Trends Cogn. Sci. 17, 502-509. https://doi.org/10.1016/j.tics.2013.08.012

Beaulieu, C., 2002. The basis of anisotropic water diffusion in the nervous system - A technical review. NMR Biomed. 15, 435-455. https://doi.org/10.1002/nbm.782

Beeri, M.S., Lee, H., Cheng, H., Wollman, D., Silverman, J.M., Prohovnik, I., 2011. Memory activation in healthy nonagenarians. Neurobiol. Aging 32, 515-523. https://doi.org/10.1016/j.neurobiolaging.2009.02.022

Bennett, I.J., Greenia, D.E., Maillard, P., Sajjadi, S.A., DeCarli, C., Corrada, M.M., Kawas, C.H., 2017. Age-related white matter integrity differences in oldest-old without dementia. Neurobiol. Aging 56, 108-114. https://doi.org/10.1016/J.NEUROBIOLAGING.2017.04.013

Bookheimer, S.Y., Salat, D.H., Terpstra, M., Ances, B.M., Barch, D.M., Buckner, R.L., Burgess, G.C., Curtiss, S.W., Diaz-Santos, M., Elam, J.S., Fischl, B., Greve, D.N., Hagy, H.A., Harms, M.P., Hatch, O.M., Hedden, T., Hodge, C., Japardi, K.C., Kuhn, T.P., Ly, T.K., Smith, S.M., Somerville, L.H., Uğurbil, K., van der Kouwe, A., Van Essen, D., Woods, R.P., Yacoub, E., 2019. The Lifespan Human Connectome Project in Aging: An overview. Neuroimage 185, 335-348. https://doi.org/10.1016/j.neuroimage.2018.10.009

Braak, H., Braak, E., 1997. Frequency of stages of Alzheimer-related lesions in different age categories. Neurobiol. Aging 18, 351-357. https://doi.org/10.1016/S0197-4580(97)00056-0

Brickman, A.M., Schupf, N., Manly, J.J., Luchsinger, J.A., Andrews, H., Tang, M.X., Reitz, C., Small, S.A., Mayeux, R., Decarli, C., Brown, T.R., 2008. Brain morphology in older African Americans, caribbean hispanics, and whites from northern Manhattan. Arch. Neurol. 65, 1053-1061. https://doi.org/10.1001/archneur.65.8.1053

Brookmeyer, R., Abdalla, N., Kawas, C.H., Corrada, M.M., 2017. Forecasting the prevalence of preclinical and clinical Alzheimer's disease in the United States. Alzheimers. Dement. 0. https://doi.org/10.1016/j.jalz.2017.10.009

Cabeza, R., 2002. Hemispheric asymmetry reduction in older adults: The HAROLD model. 
Psychol. Aging 17, 85-100. https://doi.org/10.1037/0882-7974.17.1.85

Cabeza, R., Albert, M., Belleville, S., Craik, F.I.M., Duarte, A., Grady, C.L., Lindenberger, U., Nyberg, L., Park, D.C., Reuter-Lorenz, P.A., Rugg, M.D., Steffener, J., Rajah, M.N., 2018. Maintenance, reserve and compensation: the cognitive neuroscience of healthy ageing. Nat. Rev. Neurosci. 19, 701-710. https://doi.org/10.1038/s41583-018-0068-2

Cabeza, R., Dennis, N.A., 2014. Frontal Lobes and Aging. Princ. Front. Lobe Funct. 628-652. https://doi.org/10.1093/med/9780199837755.003.0044

Cabeza, R., Grady, C.L., Nyberg, L., McIntosh, A.R., Tulving, E., Kapur, S., Jennings, J.M., Houle, S., Craik, F.I.M., 1997. Age-Related Differences in Neural Activity during Memory Encoding and Retrieval: A Positron Emission Tomography Study. J. Neurosci. 17, 391-400. https://doi.org/10.1523/JNEUROSCI.17-01-00391.1997

Carmichael, O., Mungas, D., Beckett, L., Harvey, D., Tomaszewski Farias, S., Reed, B., Olichney, J., Miller, J., DeCarli, C., 2012. MRI predictors of cognitive change in a diverse and carefully characterized elderly population. Neurobiol. Aging 33, 83-95.e2. https://doi.org/10.1016/J.NEUROBIOLAGING.2010.01.021

Carr, V.A., Bernstein, J.D., Favila, S.E., Rutt, B.K., Kerchner, G.A., Wagner, A.D., 2017. Individual differences in associative memory among older adults explained by hippocampal subfield structure and function. Proc. Natl. Acad. Sci. U. S. A. 114, 12075-12080. https://doi.org/10.1073/pnas.1713308114

Cole, D.M., Smith, S.M., Beckmann, C.F., 2010. Advances and pitfalls in the analysis and interpretation of resting-state FMRI data. Front. Syst. Neurosci. 0, 8. https://doi.org/10.3389/FNSYS.2010.00008

Corrada, M.M., Brookmeyer, R., Berlau, D., Paganini-Hill, A., Kawas, C.H., 2008. Prevalence of dementia after age 90: Results from the 90+ study. Neurology 71, 337-343. https://doi.org/10.1212/01.wnl.0000310773.65918.cd

Corrada, M.M., Brookmeyer, R., Paganini-Hill, A., Berlau, D., Kawas, C.H., 2010. Dementia Incidence Continues to Increase with Age in the Oldest Old The 90+ Study. Ann. Neurol. 67, 114. https://doi.org/10.1002/ANA.21915

Craik, F.I.M., Rose, N.S., 2012. Memory encoding and aging: A neurocognitive perspective. Neurosci. Biobehav. Rev. 36, 1729-1739. https://doi.org/10.1016/j.neubiorev.2011.11.007

Davis, S.W., Dennis, N.A., Buchler, N.G., White, L.E., Madden, D.J., Cabeza, R., 2009. Assessing the effects of age on long white matter tracts using diffusion tensor tractography. Neuroimage 46, 530-541. https://doi.org/10.1016/j.neuroimage.2009.01.068

Davis, S.W., Dennis, N.A., Daselaar, S.M., Fleck, M.S., Cabeza, R., 2008. Qué PASA? the posterior-anterior shift in aging. Cereb. Cortex 18, 1201-1209. https://doi.org/10.1093/cercor/bhm155

De Leeuw, F.E., De Groot, J.C., Achten, E., Oudkerk, M., Ramos, L.M.P., Heijboer, R., Hofman, A., Jolles, J., Van Gijn, J., Breteler, M.M.B., 2001. Prevalence of cerebral white matter lesions in elderly people: A population based magnetic resonance imaging study. The Rotterdam Scan Study. J. Neurol. Neurosurg. Psychiatry 70, 9-14. https://doi.org/10.1136/jnnp.70.1.9

Eavani, H., Habes, M., Satterthwaite, T.D., An, Y., Hsieh, M.-K., Honnorat, N., Erus, G., Doshi, J., Ferrucci, L., Beason-Held, L.L., Resnick, S.M., Davatzikos, C., 2018. Heterogeneity of structural and functional imaging patterns of advanced brain aging revealed via machine learning methods. Neurobiol. Aging 71, 41. https://doi.org/10.1016/J.NEUROBIOLAGING.2018.06.013 
Eguchi, Y., Noda, Y., Nakajima, S., Tsugawa, S., Kida, H., Plitman, E., Graff-Guerrero, A., Chakravarty, M.M., Takayama, M., Arai, Y., Matsuda, H., Mimura, M., Niimura, H., 2019. Subiculum volumes associated with memory function in the oldest-old individuals aged 95 years and older. Geriatr. Gerontol. Int. 19, 347-351. https://doi.org/10.1111/ggi.13614

Fjell, A.M., McEvoy, L., Holland, D., Dale, A.M., Walhovd, K.B., 2014. What is normal in normal aging? Effects of Aging, Amyloid and Alzheimer's Disease on the Cerebral Cortex and the Hippocampus. Prog. Neurobiol. 117, 20. https://doi.org/10.1016/J.PNEUROBIO.2014.02.004

Gardner, R.C., Valcour, V., Yaffe, K., 2013. Dementia in the oldest old: A multi-factorial and growing public health issue. Alzheimer's Res. Ther. https://doi.org/10.1186/alzrt181

Gorbach, T., Pudas, S., Lundquist, A., Orädd, G., Josefsson, M., Salami, A., de Luna, X., Nyberg, L., 2017. Longitudinal association between hippocampus atrophy and episodicmemory decline. Neurobiol. Aging 51, 167-176. https://doi.org/10.1016/j.neurobiolaging.2016.12.002

Gosche, K., Mortimer, J., Smith, C., Markesbery, W., Snowdon, D., 2002. Hippocampal volume as an index of Alzheimer neuropathology. Neurology 58, 1476-1482.

Grady, C.L., 2008. Cognitive neuroscience of aging. Ann. N. Y. Acad. Sci. 1124, 127-144. https://doi.org/10.1196/annals.1440.009

Greve, D.N., Fischl, B., 2009. Accurate and Robust Brain Image Alignment using Boundarybased Registration. Neuroimage 48, 63. https://doi.org/10.1016/J.NEUROIMAGE.2009.06.060

Grinband, J., Steffener, J., Razlighi, Q.R., Stern, Y., 2017. BOLD neurovascular coupling does not change significantly with normal aging. Hum. Brain Mapp. 38, 3538. https://doi.org/10.1002/HBM.23608

Haacke, E.M., Cheng, N.Y.C., House, M.J., Liu, Q., Neelavalli, J., Ogg, R.J., Khan, A., Ayaz, M., Kirsch, W., Obenaus, A., 2005. Imaging iron stores in the brain using magnetic resonance imaging. Magn. Reson. Imaging 1-25. https://doi.org/10.1016/j.mri.2004.10.001

Hallgren, B., Sourander, P., 1958. THE EFFECT OF AGE ON THE NON-HAEMIN IRON IN THE HUMAN BRAIN. J. Neurochem. 3, 41-51. https://doi.org/10.1111/j.14714159.1958.tb12607.x

Hartel, C.R., Buckner, R.L., 2006. Utility of Brain Imaging Methods in Research on Aging.

Haynes, B.I., Bunce, D., Kochan, N.A., Wen, W., Brodaty, H., Sachdev, P.S., 2017. Associations between reaction time measures and white matter hyperintensities in very old age. Neuropsychologia 96, 249-255. https://doi.org/10.1016/j.neuropsychologia.2017.01.021

He, W., Muenchrath, M.N., 2011. 90+ in the United States: 2006-2008 American Community Survey Reports.

Head, D., Buckner, R.L., Shimony, J.S., Williams, L.E., Akbudak, E., Conturo, T.E., McAvoy, M., Morris, J.C., Snyder, A.Z., 2004. Differential Vulnerability of Anterior White Matter in Nondemented Aging with Minimal Acceleration in Dementia of the Alzheimer Type: Evidence from Diffusion Tensor Imaging. Cereb. Cortex 14, 410-423. https://doi.org/10.1093/cercor/bhh003

Head, D., Snyder, A.Z., Girton, L.E., Morris, J.C., Buckner, R.L., 2005. Frontal-hippocampal double dissociation between normal aging and Alzheimer's disease. Cereb. Cortex 15, 732739. https://doi.org/10.1093/cercor/bhh174

Hedden, T., Gabrieli, J.D.E., 2005. Healthy and pathological processes in adult development: New evidence from neuroimaging of the aging brain. Curr. Opin. Neurol. 18, 740-747. 
https://doi.org/10.1097/01.wco.0000189875.29852.48

Hedden, T., Gabrieli, J.D.E., 2004. Insights into the ageing mind: a view from cognitive neuroscience. Nat. Rev. Neurosci. 5, 87-96. https://doi.org/10.1038/nrn1323

Holland, D., Desikan, R.S., Dale, A.M., McEvoy, L.K., 2012. Rates of decline in Alzheimer disease decrease with age. PLoS One 7. https://doi.org/10.1371/journal.pone.0042325

Huppert, T.J., Karim, H., Lin, C.C., Alqahtani, B.A., Greenspan, S.L., Sparto, P.J., 2017. Functional imaging of cognition in an old-old population: A case for portable functional near-infrared spectroscopy. PLoS One 12, e0184918. https://doi.org/10.1371/journal.pone.0184918

Jagust, W., 2013. Vulnerable neural systems and the borderland of brain aging and neurodegeneration. Neuron 77, 219-34. https://doi.org/10.1016/j.neuron.2013.01.002

Jernigan, T.L., Archibald, S.L., Fennema-Notestine, C., Gamst, A.C., Stout, J.C., Bonner, J., Hesselink, J.R., 2001. Effects of age on tissues and regions of the cerebrum and cerebellum. Neurobiol. Aging 22, 581-594. https://doi.org/10.1016/S0197-4580(01)00217-2

Jiang, J., Liu, T., Crawford, J.D., Kochan, N.A., Brodaty, H., Sachdev, P.S., Wen, W., 2020. Stronger bilateral functional connectivity of the frontoparietal control network in nearcentenarians and centenarians without dementia. Neuroimage 215, 116855. https://doi.org/10.1016/j.neuroimage.2020.116855

Jones, D.K., 2008. Studying connections in the living human brain with diffusion MRI. Cortex 44, 936-952. https://doi.org/10.1016/J.CORTEX.2008.05.002

Kannurpatti, S.S., Motes, M.A., Rypma, B., Biswal, B.B., 2010. Neural and vascular variability and the fMRI-BOLD response in normal aging. Magn. Reson. Imaging 28, 466-476. https://doi.org/10.1016/j.mri.2009.12.007

Kawas, C., Corrada, M., 2006. Alzheimers and Dementia in the Oldest-Old: A Century of Challenges. Curr. Alzheimer Res. 3, 411-419. https://doi.org/10.2174/156720506779025233

Kawas, C.H., Kim, R.C., Sonnen, J.A., Bullain, S.S., Trieu, T., Corrada, M.M., 2015. Multiple pathologies are common and related to dementia in the oldest-old: The $90+$ Study. Neurology 85, 535-542. https://doi.org/10.1212/WNL.0000000000001831

Kawas, C.H., Legdeur, N., Corrada, M.M., 2021. What have we learned from cognition in the oldest-old. Curr. Opin. Neurol. 34, 258-265. https://doi.org/10.1097/WCO.0000000000000910

Kennedy, K.M., Rodrigue, K.M., Bischof, G.N., Hebrank, A.C., Reuter-Lorenz, P.A., Park, D.C., 2015. Age trajectories of functional activation under conditions of low and high processing demands: an adult lifespan fMRI study of the aging brain. Neuroimage 104, $21-$ 34. https://doi.org/10.1016/j.neuroimage.2014.09.056

Langnes, E., Sneve, M.H., Sederevicius, D., Amlien, I.K., Walhovd, K.B., Fjell, A.M., 2020. Anterior and posterior hippocampus macro- and microstructure across the lifespan in relation to memory_A longitudinal study. Hippocampus hipo.23189.

https://doi.org/10.1002/hipo.23189

Laukka, E.J., Lövdén, M., Kalpouzos, G., Li, T.Q., Jonsson, T., Wahlund, L.O., Fratiglioni, L., Bäckman, L., 2013. Associations between white matter microstructure and cognitive performance in old and very old age. PLoS One 8, e81419.

https://doi.org/10.1371/journal.pone.0081419

Legdeur, N., Badissi, M., Yaqub, M., Beker, N., Sudre, C.H., ten Kate, M., Gordon, M.F., Novak, G., Barkhof, F., van Berckel, B.N.M., Holstege, H., Muller, M., Scheltens, P., 
Maier, A.B., Visser, P.J., 2020. What determines cognitive functioning in the oldest-old? The EMIF-AD 90+ Study. Journals Gerontol. Ser. B. https://doi.org/10.1093/geronb/gbaa152

Legdeur, N., Visser, P.J., Woodworth, D.C., Muller, M., Fletcher, E., Maillard, P., Scheltens, P., DeCarli, C., Kawas, C.H., Corrada, M.M., 2019. White Matter Hyperintensities and Hippocampal Atrophy in Relation to Cognition: The 90+ Study. J. Am. Geriatr. Soc. jgs.15990. https://doi.org/10.1111/jgs.15990

Li, Q., Dong, C., Liu, T., Chen, X., Perry, A., Jiang, J., Cheng, J., Niu, H., Kochan, N.A., Brodaty, H., Sachdev, P.S., Wen, W., 2020. Longitudinal Changes in Whole-Brain Functional Connectivity Strength Patterns and the Relationship With the Global Cognitive Decline in Older Adults. Front. Aging Neurosci. 12, 1-10. https://doi.org/10.3389/fnagi.2020.00071

Liu, G., Allen, B., Lopez, O., Aizenstein, H., Boudreau, R., Newman, A., Yaffe, K., Kritchevsky, S., Launer, L., Satterfield, S., Simonsick, E., Rosano, C., 2015. Racial Differences in Gray Matter Integrity by Diffusion Tensor in Black and White Octogenarians. Curr. Alzheimer Res. 12, 648-654. https://doi.org/10.2174/1567205011666141107153634

Lockhart, S.N., DeCarli, C., 2014. Structural Imaging Measures of Brain Aging. Neuropsychol. Rev. 24, 271. https://doi.org/10.1007/S11065-014-9268-3

Logothetis, N.K., 2008. What we can do and what we cannot do with fMRI. Nature 453, 869-78. https://doi.org/10.1038/nature06976

Lopez, O.L., Klunk, W.E., Mathis, C., Coleman, R.L., Price, J., Becker, J.T., Aizenstein, H.J., Snitz, B., Cohen, A., Ikonomovic, M., McDade, E., Dekosky, S.T., Weissfeld, L., Kuller, L.H., 2014. Amyloid, neurodegeneration, and small vessel disease as predictors of dementia in the oldest-old. Neurology 83, 1804-1811. https://doi.org/10.1212/WNL.0000000000000977

Lövdén, M., Köhncke, Y., Laukka, E.J., Kalpouzos, G., Salami, A., Li, T.Q., Fratiglioni, L., Bäckman, L., 2014. Changes in perceptual speed and white matter microstructure in the corticospinal tract are associated in very old age. Neuroimage 102, 520-530. https://doi.org/10.1016/j.neuroimage.2014.08.020

Lövdén, M., Laukka, E.J., Rieckmann, A., Goria Kalpouzos, G., Li, T.-Q., Jonsson, T., Wahlund, L.-O., Fratiglioni, L., Bäckman, L., Lövdén, M., 2013. The Dimensionality of BetweenPerson Differences in White Matter Microstructure in Old Age. Hum. Brain Mapp. 34, 1386-1398. https://doi.org/10.1002/hbm.21518

Madden, D.J., Bennett, I.J., Song, A.W., 2009. Cerebral white matter integrity and cognitive aging: contributions from diffusion tensor imaging. Neuropsychol. Rev. 19, 415-35. https://doi.org/10.1007/s11065-009-9113-2

Merenstein, J.L., Corrada, M.M., Kawas, C.H., Bennett, I.J., 2021. Age affects white matter microstructure and episodic memory across the older adult lifespan. Neurobiol. Aging 106, 282-291. https://doi.org/10.1016/j.neurobiolaging.2021.06.021

Montine, T.J., Cholerton, B.A., Corrada, M.M., Edland, S.D., Flanagan, M.E., Hemmy, L.S., Kawas, C.H., White, L.R., 2019. Concepts for brain aging: Resistance, resilience, reserve, and compensation. Alzheimer's Res. Ther. https://doi.org/10.1186/s13195-019-0479-y

Mori, S., Zhang, J., 2006. Principles of Diffusion Tensor Imaging and Its Applications to Basic Neuroscience Research. Neuron 51, 527-539.

https://doi.org/10.1016/J.NEURON.2006.08.012

Mueller, E.A., Moore, M.M., Kerr, D.C.R., Sexton, G., Camicioli, R.M., Howieson, D.B., 
Quinn, J.F., Kaye, J.A., 1998. Brain volume preserved in healthy elderly through the eleventh decade. Neurology 51, 1555-1562. https://doi.org/10.1212/WNL.51.6.1555

Nyberg, L., Lövdén, M., Riklund, K., Lindenberger, U., Bäckman, L., 2012. Memory aging and brain maintenance. Trends Cogn. Sci. 16, 292-305. https://doi.org/10.1016/J.TICS.2012.04.005

Nyberg, L., Pudas, S., 2018. Successful Memory Aging. Annu. Rev. Psychol. 70, 219-243. https://doi.org/10.1146/annurev-psych-010418-103052

O’Shea, A., Cohen, R.A., Porges, E.C., Nissim, N.R., Woods, A.J., 2016. Cognitive Aging and the Hippocampus in Older Adults. Front. Aging Neurosci. 8, 298. https://doi.org/10.3389/fnagi.2016.00298

Paolacci, L., Giannandrea, D., Mecocci, P., Parnetti, L., 2017. Biomarkers for Early Diagnosis of Alzheimer's Disease in the Oldest Old: Yes or No? J. Alzheimer's Dis. 58, 323-335. https://doi.org/10.3233/JAD-161127

Papenberg, G., Lövdén, M., Laukka, E.J., Kalpouzos, G., Keller, L., Graff, C., Köhncke, Y., Li, T.Q., Fratiglioni, L., Bäckman, L., 2015. Magnified effects of the COMT gene on whitematter microstructure in very old age. Brain Struct. Funct. 220, 2927-2938. https://doi.org/10.1007/s00429-014-0835-4

Pelkmans, W., Legdeur, N., Kate, M., Barkhof, F., Yaqub, M.M., Holstege, H., Berckel, B.N.M. Van, Scheltens, P., Flier, W.M. Van Der, Visser, P.J., Tijms, B.M., 2021. Amyloid- b , cortical thickness, and subsequent cognitive decline in cognitively normal oldest-old. Ann. Clin. Transl. Neurol. 1-11. https://doi.org/10.1002/acn3.51273

Peltz, C.B., Corrada, M.M., Berlau, D.J., Kawas, C.H., 2011. Incidence of dementia in oldest-old with amnestic MCI and other cognitive impairments. Neurology 77, 1906-1912. https://doi.org/10.1212/WNL.0b013e318238ee89

Peters, A., 2002. The effects of normal aging on myelin and nerve fibers: A review. J. Neurocytol. https://doi.org/10.1023/A:1025731309829

Pfefferbaum, A., Adalsteinsson, E., Sullivan, E. V., 2005. Frontal circuitry degradation marks healthy adult aging: Evidence from diffusion tensor imaging. Neuroimage 26, 891-899. https://doi.org/10.1016/j.neuroimage.2005.02.034

Pierce, A.L., Kawas, C.H., 2017. Dementia in the oldest old: Beyond Alzheimer disease. PLoS Med. 14, e1002263. https://doi.org/10.1371/journal.pmed.1002263

Piguet, O., Ridley, L., Grayson, D.A., Bennett, H.P., Creasey, H., Lye, T.C., Anthony Broe, G., 2003. Are MRI White Matter Lesions Clinically Significant in the "Old-Old"? Evidence from the Sydney Older Persons Study. Orig. Res. Artic. Dement Geriatr Cogn Disord 15, 143-150. https://doi.org/10.1159/000068482

Polvikoski, T.M., Van Straaten, E.C.W., Barkhof, F., Sulkava, R., Aronen, H.J., Niinistö, L., Oinas, M., Scheltens, P., Erkinjuntti, T., Kalaria, R.N., 2010. Frontal lobe white matter hyperintensities and neurofibrillary pathology in the oldest old. Neurology.

Poon, L.W., Jazwinski, M., Green, R.C., Woodard, J.L., Martin, P., Rodgers, W.L., Johnson, M.A., Hausman, D., Arnold, J., Davey, A., Batzer, M.A., Markesbery, W.R., Gearing, M., Siegler, I.C., Reynolds, S., Dai, J., 2007. Methodological Considerations in Studying Centenarians: Lessons Learned From the Georgia Centenarian Studies. Annu. Rev. Gerontol. Geriatr. 27, 231-264.

Poulakis, K., Reid, R.I., Przybelski, S.A., Knopman, D.S., Graff-Radford, J., Lowe, V.J., Mielke, M.M., Machulda, M.M., Jack, C.R., Petersen, R.C., Westman, E., Vemuri, P., 2021. Longitudinal deterioration of white-matter integrity: heterogeneity in the ageing population. 
Brain Commun. 3. https://doi.org/10.1093/braincomms/fcaa238

Prawiroharjo, P., Yamashita, Ken ichiro, Yamashita, Koji, Togao, O., Hiwatashi, A., Yamasaki, R., Kira, J. ichi, 2020. Disconnection of the right superior parietal lobule from the precuneus is associated with memory impairment in oldest-old Alzheimer's disease patients. Heliyon 6, e04516. https://doi.org/10.1016/j.heliyon.2020.e04516

Raz, N., Ghisletta, P., Rodrigue, K.M., Kennedy, K.M., Lindenberger, U., 2010. Trajectories of brain aging in middle-aged and older adults: Regional and individual differences. Neuroimage 51, 501-511. https://doi.org/10.1016/J.NEUROIMAGE.2010.03.020

Raz, N., Lindenberger, U., Rodrigue, K.M., Kennedy, K.M., Head, D., Williamson, A., Dahle, C., Gerstorf, D., Acker, J.D., 2005. Regional Brain Changes in Aging Healthy Adults: General Trends, Individual Differences and Modifiers. Cereb. Cortex 15, 1676-1689. https://doi.org/10.1093/cercor/bhi044

Reas, E.T., Hagler, D.J., Zhong, A.J., Lee, R.R., Dale, A.M., Linda, K., 2021. Brain microstructure mediates sex-specific patterns of cognitive aging 13, 1-21.

Resnick, S.M., Pham, D.L., Kraut, M.A., Zonderman, A.B., Davatzikos, C., Kapur, S., Jennings, J., Houle, S., Craik, F., 2003. Longitudinal magnetic resonance imaging studies of older adults: a shrinking brain. J. Neurosci. 23, 3295-301. https://doi.org/10.1523/jneurosci.229507.2008

Reuter-Lorenz, P.A., Cappell, K.A., 2008. Neurocognitive Aging and the Compensation Hypothesis. Curr. Dir. Psychol. Sci.

Rosa, G., Giannotti, C., Martella, L., Massa, F., Serafini, G., Pardini, M., Nobili, F.M., Monacelli, F., Mecocci, P., 2020. Brain Aging, Cardiovascular Diseases, Mixed Dementia, and Frailty in the Oldest Old: From Brain Phenotype to Clinical Expression. J. Alzheimer's Dis. 75, 1083-1103. https://doi.org/10.3233/JAD-191075

Rosano, C., Abebe, K.Z., Aizenstein, H.J., Boudreau, R., Jennings, J.R., Venkatraman, V., Harris, T.B., Yaffe, K., Satterfield, S., Newman, A.B., 2015. Longitudinal systolic blood pressure characteristics and integrity of white matter tracts in a cohort of very old black and white adults. Am. J. Hypertens. 28, 326-334. https://doi.org/10.1093/ajh/hpu134

Rosano, C., Aizenstein, H., Cochran, J., Saxton, J., De Kosky, S., Newman, A.B., Kuller, L.H., Lopez, O.L., Carter, C.S., 2005a. Functional neuroimaging indicators of successful executive control in the oldest old. Neuroimage 28, 881-889. https://doi.org/10.1016/j.neuroimage.2005.05.059

Rosano, C., Aizenstein, H.J., Cochran, J.L., Saxton, J.A., De Kosky, S.T., Newman, A.B., Kuller, L.H., Lopez, O.L., Carter, C.S., 2005b. Event-related functional magnetic resonance imaging investigation of executive control in very old individuals with mild cognitive impairment. Biol. Psychiatry 57, 761-767. https://doi.org/10.1016/j.biopsych.2004.12.031

Rosano, C., Aizenstein, H.J., Newman, A.B., Venkatraman, V., Harris, T., Ding, J., Satterfield, S., Yaffe, K., 2012. Neuroimaging differences between older adults with maintained versus declining cognition over a 10-year period. Neuroimage 62, 307-313. https://doi.org/10.1016/j.neuroimage.2012.04.033

Ruetten, P.P.R., Gillard, J.H., Graves, M.J., 2019. Introduction to Quantitative Susceptibility Mapping and Susceptibility Weighted Imaging. Br. J. Radiol. 92. https://doi.org/10.1259/BJR.20181016

Salat, D.H., 2011. The declining infrastructure of the aging brain. Brain Connect. 1, 279-93. https://doi.org/10.1089/brain.2011.0056

Salat, D.H., Kaye, J.A., Janowsky, J.S., 1999. Prefrontal gray and white matter volumes in 
healthy aging and Alzheimer disease. Arch. Neurol. 56, 338-344.

https://doi.org/10.1001/archneur.56.3.338

Salat, D.H., Lee, S.Y., van der Kouwe, A.J., Greve, D.N., Fischl, B., Rosas, H.D., 2009. Ageassociated alterations in cortical gray and white matter signal intensity and gray to white matter contrast. Neuroimage 48, 21-28. https://doi.org/10.1016/j.neuroimage.2009.06.074

Scheller, E., Minkova, L., Leitner, M., Klöppel, S., 2014. Attempted and successful compensation in preclinical and early manifest neurodegeneration - a review of task fMRI studies. Front. Psychiatry 5, 132. https://doi.org/10.3389/FPSYT.2014.00132/BIBTEX

Simonsick, E.M., Group, for the H.A.S., Newman, A.B., Group, for the H.A.S., Nevitt, M.C., Group, for the H.A.S., Kritchevsky, S.B., Group, for the H.A.S., Ferrucci, L., Group, for the H.A.S., Guralnik, J.M., Group, for the H.A.S., Harris, T., Group, for the H.A.S., 2001. Measuring Higher Level Physical Function in Well-Functioning Older AdultsExpanding Familiar Approaches in the Health ABC Study. Journals Gerontol. Ser. A 56, M644-M649. https://doi.org/10.1093/GERONA/56.10.M644

Stebbins, G.T., Murphy, C.M., 2009. Diffusion tensor imaging in Alzheimer's disease and mild cognitive impairment. Behav. Neurol. https://doi.org/10.3233/BEN-2009-0234

Stern, Y., 2006. Cognitive reserve and Alzheimer disease. Alzheimer Dis. Assoc. Disord. 20, 112-117. https://doi.org/10.1097/01.WAD.0000213815.20177.19

Stickel, A., Kawa, K., Walther, K., Glisky, E., Richholt, R., Huentelman, M., Ryan, L., 2018. Age-modulated associations between KIBRA, brain volume, and verbal memory among healthy older adults. Front. Aging Neurosci. 9. https://doi.org/10.3389/fnagi.2017.00431

Stricker, N.H., Chang, Y.L., Fennema-Notestine, C., Delano-Wood, L., Salmon, D.P., Bondi, M.W., Dale, A.M., 2011. Distinct profiles of brain and cognitive changes in the very old with Alzheimer disease. Neurology 77, 713-721. https://doi.org/10.1212/WNL.0b013e31822b0004

Tang, H., Liu, T., Liu, H., Jiang, J., Cheng, J., Niu, H., Li, S., Brodaty, H., Sachdev, P., Wen, W., 2021. A slower rate of sulcal widening in the brains of the nondemented oldest old. Neuroimage 229, 117740. https://doi.org/10.1016/j.neuroimage.2021.117740

Tanskanen, M., Kalaria, R., Notkola, I.-L., Mäkelä, M., Polvikoski, T., Myllykangas, L., Sulkava, R., Kalimo, H., Paetau, A., Scheltens, P., Barkhof, F., ECW, van, Erkinjuntti, T., 2013. Relationships Between White Matter Hyperintensities, Cerebral Amyloid Angiopathy and Dementia in a Population-based Sample of the Oldest Old. Curr. Alzheimer Res. 10, 1090-1097. https://doi.org/10.2174/15672050113106660177

Tarantini, S., Tran, C.H.T., Gordon, G.R., Ungvari, Z., Csiszar, A., 2017. Impaired neurovascular coupling in aging and Alzheimer's disease: contribution of astrocyte dysfunction and endothelial impairment to cognitive decline. Exp. Gerontol. 94, 52. https://doi.org/10.1016/J.EXGER.2016.11.004

Tromp, D., Dufour, A., Lithfous, S., Pebayle, T., Després, O., 2015. Episodic memory in normal aging and Alzheimer disease: Insights from imaging and behavioral studies. Ageing Res. Rev. 24, 232-262. https://doi.org/10.1016/J.ARR.2015.08.006

Tucker, A.M., Stern, Y., 2011. Cognitive reserve in aging. Curr. Alzheimer Res. 8, 354-60. https://doi.org/10.2174/156720511795745320

van Bergen, J.M.G., Li, X., Quevenco, F.C., Gietl, A.F., Treyer, V., Leh, S.E., Meyer, R., Buck, A., Kaufmann, P.A., Nitsch, R.M., van Zijl, P.C.M., Hock, C., Unschuld, P.G., 2018. Low cortical iron and high entorhinal cortex volume promote cognitive functioning in the oldestold. Neurobiol. Aging 64, 68-75. https://doi.org/10.1016/j.neurobiolaging.2017.12.014 
Venkatesh, A., Daugherty, A.M., Bennett, I.J., 2021. Neuroimaging measures of iron and gliosis explain memory performance in aging. Hum. Brain Mapp. https://doi.org/10.1002/HBM.25652

Venkatraman, V.K., Aizenstein, H.J., Newman, A.B., Yaffe, K., Harris, T., Kritchevsky, S., Ayonayon, H.N., Rosano, C., 2011. Lower digit symbol substitution score in the oldest old is related to magnetization transfer and diffusion tensor imaging of the white matter. Front. Aging Neurosci. 3, 1-8. https://doi.org/10.3389/fnagi.2011.00011

Vidal-Piñeiro, D., Sneve, M.H., Nyberg, L.H., Mowinckel, A.M., Sederevicius, D., Walhovd, K.B., Fjell, A.M., 2018. Maintained Frontal Activity Underlies High Memory Function Over 8 Years in Aging. Cereb. Cortex 1-13. https://doi.org/10.1093/cercor/bhy177

Von Gunten, A., Ebbing, K., Imhof, A., Giannakopoulos, P., Kövari, E., 2010. Brain aging in the oldest-old. Curr. Gerontol. Geriatr. Res. 2010. https://doi.org/10.1155/2010/358531

Wang, T.H., Kruggel, F., Rugg, M.D., 2009. Effects of advanced aging on the neural correlates of successful recognition memory. Neuropsychologia 47, 1352-61. https://doi.org/10.1016/j.neuropsychologia.2009.01.030

Wardlaw, J.M., Valdés Hernández, M.C., Muñoz-Maniega, S., 2015. What are white matter hyperintensities made of? Relevance to vascular cognitive impairment. J. Am. Heart Assoc. https://doi.org/10.1161/JAHA.114.001140

West, K.L., Zuppichini, M.D., Turner, M.P., Sivakolundu, D.K., Zhao, Y., Abdelkarim, D., Spence, J.S., Rypma, B., 2019. BOLD hemodynamic response function changes significantly with healthy aging. Neuroimage 188, 198-207. https://doi.org/10.1016/j.neuroimage.2018.12.012

West, R.L., 1996. An application of prefrontal cortex function theory to cognitive aging.

Psychol. Bull. 120, 272-292. https://doi.org/10.1037/0033-2909.120.2.272

Wollman, D.E., Beeri, M.S., Weinberger, M., Cheng, H., Silverman, J.M., Prohovnik, I., 2004. Tolerance of MRI procedures by the oldest old. Magn. Reson. Imaging 22, 1299-1304. https://doi.org/10.1016/J.MRI.2004.08.009

Woodworth, D.C., Scambray, K.A., Corrada, M.M., Kawas, C.H., Ahmad Sajjadi, S., 2021. Neuroimaging in the Oldest-Old: A Review of the Literature. J. Alzheimer's Dis. 1-19. https://doi.org/10.3233/jad-201578

Yang, Z., Slavin, M.J., Sachdev, P.S., 2013. Dementia in the oldest old. Nat. Rev. Neurol. https://doi.org/10.1038/nrneurol.2013.105

Yang, Z., Wen, W., Jiang, J., Crawford, J.D., Reppermund, S., Levitan, C., Slavin, M.J., Kochan, N.A., Richmond, R.L., Brodaty, H., Trollor, J.N., Sachdev, P.S., 2016a. Age-associated differences on structural brain MRI in nondemented individuals from 71 to 103 years. Neurobiol. Aging 40, 86-97. https://doi.org/10.1016/j.neurobiolaging.2016.01.006

Yang, Z., Wen, W., Jiang, J., D. Crawford, J., Reppermund, S., Levitan, C., J. Slavin, M., A. Kochan, N., L. Richmond, R., Brodaty, H., N. Trollor, J., S. Sachdev, P., 2016b. Structural MRI Biomarkers of Mild Cognitive Impairment from Young Elders to Centenarians. Curr. Alzheimer Res. 13, 256-267. https://doi.org/10.2174/1567205013666151218150534

Young, P.N.E., Estarellas, M., Coomans, E., Srikrishna, M., Beaumont, H., Maass, A., Venkataraman, A. V., Lissaman, R., Jiménez, D., Betts, M.J., McGlinchey, E., Berron, D., O’Connor, A., Fox, N.C., Pereira, J.B., Jagust, W., Carter, S.F., Paterson, R.W., Schöll, M., 2020. Imaging biomarkers in neurodegeneration: current and future practices. Alzheimer's Res. Ther. 2020121 12, 1-17. https://doi.org/10.1186/S13195-020-00612-7

Zamboni, G., Griffanti, L., Mazzucco, S., Pendlebury, S.T., Rothwell, P.M., 2019. Age- 
dependent association of white matter abnormality with cognition after TIA or minor stroke. Neurology 93, E272-E282. https://doi.org/10.1212/WNL.0000000000007772

Zecca, L., Youdim, M.B.H., Riederer, P., Connor, J.R., Crichton, R.R., 2004. Iron, brain ageing and neurodegenerative disorders. Nat. Rev. Neurosci. https://doi.org/10.1038/nrn1537

Zhang, H., Schneider, T., Wheeler-Kingshott, C.A., Alexander, D.C., 2012. NODDI: Practical in vivo neurite orientation dispersion and density imaging of the human brain. Neuroimage 61, 1000-1016. https://doi.org/10.1016/j.neuroimage.2012.03.072 


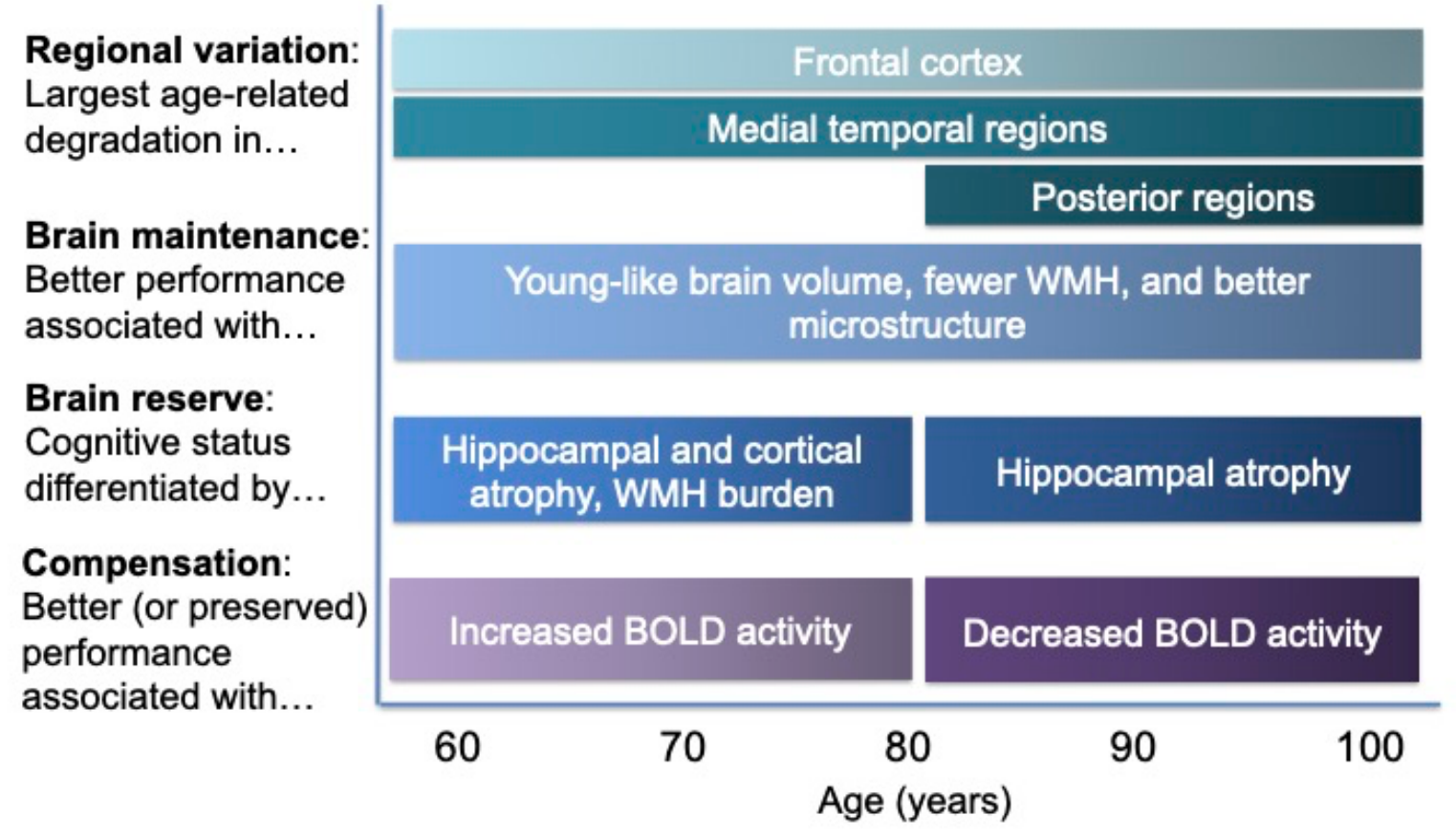

Figure 1. Neuroimaging patterns relevant to each neurocognitive aging theory reviewed here

(left) are presented separately for younger-old (ages $<80$ years) and oldest-old ( $80+$ years) adults. Brain maintenance captures similar effects across the older adult lifespan, whereas oldestold adults exhibit different patterns of regional variation, brain reserve, and functional compensation. $\mathrm{WMH}=$ white matter hyperintensities, $\mathrm{BOLD}=$ blood-oxygenation-leveldependent. 
Table 1. Summary of structural studies examining brain aging.

\begin{tabular}{|c|c|c|c|c|c|c|}
\hline \multirow[b]{2}{*}{ Author (year) } & \multirow{2}{*}{$\begin{array}{l}\text { Sample } \\
\text { age }(n)\end{array}$} & \multirow{2}{*}{$\begin{array}{l}\text { Structural } \\
\text { Modality }\end{array}$} & \multicolumn{4}{|c|}{ Age Effect(s) } \\
\hline & & & Frontal & MTL & Posterior & Whole brain \\
\hline \multicolumn{7}{|l|}{ Volumetry \& Morphometry } \\
\hline \multirow[t]{2}{*}{ Yang et al. (2016a) } & $71-103(277)$ & Volume & - & - & - & - \\
\hline & & Cortical thickness & - & - & - & \\
\hline Tang et al. (2021) & $76-103(290)$ & Sulcal width & - & - & - & \\
\hline Li et al. (2020) & $\mathrm{M}=82(34)$ & Cortical thickness & - & - & & \\
\hline Van Bergen et al. (2018) & $55-96(80)$ & Volume & & $o,-$ & - & \\
\hline Mueller et al. (1998) & $65-95(46)$ & Volume & o & - & o & - \\
\hline Brickman et al. (2008) & $M=80(769)$ & Volume & & - & & - \\
\hline Salat et al. (1999) & $65-95(66)$ & Volume & - & & & \\
\hline \multicolumn{7}{|c|}{ White matter hyperintensities \& Microstructure } \\
\hline Brickman et al. (2008) & $M=80(769)$ & WMH & & & & - \\
\hline Bennett et al. (2017) & $90-103(94)$ & $\mathrm{FA}, \mathrm{MD}, \mathrm{AD}, \mathrm{RD}$ & o & - & - & \\
\hline Piguet et al. (2003) & $81-97(114)$ & WMH & - & - & - & \\
\hline Yang et al. (2016a) & $71-103(277)$ & WMH & - & - & - & - \\
\hline Lövdén et al. (2013) & $60-87(260)$ & $\mathrm{FA}, \mathrm{MD}$ & - & - & - & \\
\hline Merenstein et al. (2021) & $65-98(108)$ & $\mathrm{FA}, \mathrm{AD}, \mathrm{RD}$ & - & -- & - & \\
\hline Lövdén et al. (2014) & $81-103(563)$ & $\Delta \mathrm{FA}, \Delta \mathrm{MD}$ & - & & - & \\
\hline Polvikoski et al. (2010) & $85-104(132)$ & WMH & - & & & \\
\hline
\end{tabular}

Notes. For each structural modality and brain region, symbols indicate observations of worse brain structure with age (-; i.e., smaller volume, more white matter hyperintensities [WMH], higher diffusivity), a larger negative effect of age in one region relative to other regions (- -), or no significant effect of age (o). Volumetry and morphometry studies are sorted by age effects in the medial temporal lobe (MTL). WMH and microstructure studies are sorted by age effects in the frontal lobe. FA $=$ fractional anisotropy, MD $=$ mean diffusivity, $\mathrm{AD}=$ axial diffusivity, $\mathrm{RD}=$ radial diffusivity, $\Delta=$ longitudinal change with age. 
Table 2. Summary of structural MRI studies examining neurocognitive aging.

\begin{tabular}{|c|c|c|c|c|c|c|c|}
\hline \multirow[t]{2}{*}{ Author (year) } & \multirow[t]{2}{*}{ Sample age $(n)$} & \multirow[t]{2}{*}{ Structural modality } & \multirow[t]{2}{*}{ Cognitive domain(s) } & \multicolumn{4}{|c|}{ Relationship to Cognition } \\
\hline & & & & Frontal & MTL & Posterior & Whole brain \\
\hline \multicolumn{8}{|l|}{ Volumetry \& Morphometry } \\
\hline Carmichael et al. (2012) & $60-95(307)$ & Volume & $\Delta$ Episodic memory & & & & - \\
\hline Legdeur et al. (2019) & $\mathrm{M}=94.3(171)$ & $\Delta$ Volume & $\Delta$ Episodic memory & & - & & \\
\hline Pelkmans et al. (2021) & $88-102(57)$ & Volume & $\Delta$ Episodic memory & & - & & \\
\hline Pelkmans et al. (2021) & $88-102(57)$ & Cortical thickness & $\Delta$ Episodic memory & - & - & & \\
\hline Eguchi et al. (2019) & $96-99(10)$ & Volume & Episodic memory & & + & & \\
\hline Langnes et al. (2020) & $4-93(1,790)$ & Volume & Episodic memory & & + & & \\
\hline Legdeur et al. (2020) & $\mathrm{M}=92.4(122)$ & Volume & Episodic memory & & + & & \\
\hline Pelkmans et al. (2021) & $88-102(57)$ & Volume & Processing speed & & + & & \\
\hline Legdeur et al. (2020) & $\mathrm{M}=92.4(122)$ & Volume & Processing speed & & + & & \\
\hline Legdeur et al. (2019) & $\mathrm{M}=94.3(171)$ & $\Delta$ Volume & $\Delta$ General cognition & & - & & \\
\hline \multicolumn{8}{|c|}{ White matter hyperintensities \& Microstructure } \\
\hline Pelkmans et al. (2021) & $88-102(57)$ & WMH & $\Delta$ Episodic memory & & & & - \\
\hline Legdeur et al. (2020) & $\mathrm{M}=92.4(122)$ & WMH & Episodic memory & & & & + \\
\hline Langnes et al. (2020) & $4-93(1,790)$ & MD & Episodic memory & & + & & \\
\hline Merenstein et al. (2021) & $65-98(108)$ & $\mathrm{FA}, \mathrm{AD}$ & Episodic memory & & + & & \\
\hline Reas et al. (2021) & $56-99(147)$ & MDI & Episodic memory & + & + & + & \\
\hline Piguet et al. (2003) & $81-97(114)$ & WMH & Episodic memory & + & & & \\
\hline Pelkmans et al. (2021) & $88-102(57)$ & WMH & Processing speed & & & & + \\
\hline Liu et al. (2015) & $79-89(283)$ & WMH, MD & Processing speed & & & & + \\
\hline Venkatraman et al. (2011) & $\mathrm{M}=83(272)$ & $\mathrm{FA}, \mathrm{MD}$ & Processing speed & & & & + \\
\hline Legdeur et al. (2020) & $\mathrm{M}=92.4(122)$ & WMH & Processing speed & & & & + \\
\hline Laukka et al. (2013) & $60-87(253)$ & $\mathrm{FA}, \mathrm{MD}$ & Processing speed & + & + & + & \\
\hline Rosano et al. (2015) & $\mathrm{M}=83(311)$ & WMH, FA & Processing speed & + & & + & \\
\hline Haynes et al. (2017) & $70-90(526)$ & WMH & Processing speed & + & & & \\
\hline Lövdén et al. (2014) & $81-103(563)$ & $\Delta \mathrm{FA}, \Delta \mathrm{MD}$ & $\Delta$ Processing speed & - & & & \\
\hline Legdeur et al. (2019) & $\mathrm{M}=94.3(171)$ & $\Delta \mathrm{WMH}$ & $\Delta$ General cognition & & & & - \\
\hline Carmichael et al. (2012) & $60-95(307)$ & WMH & $\Delta$ Semantic memory & & & & - \\
\hline Carmichael et al. (2012) & $60-95(307)$ & WMH & $\Delta$ Executive function & & & & - \\
\hline
\end{tabular}

Notes. For each structural modality and brain region, symbols indicate positive (+) or negative (-) associations between brain structure (i.e., smaller volume, more white matter hyperintensities [WMH], higher diffusivity) and cognitive performance. Processing speed was reverse coded with higher values representing better performance. All studies are sorted by the cognitive domain examined. FA = fractional anisotropy, $\mathrm{MD}=$ mean diffusivity, $\mathrm{AD}=$ axial diffusivity, $\Delta=$ longitudinal change, $\mathrm{MTL}=$ medial temporal lobe, $\mathrm{MDI}=$ multicompartment diffusion imaging. 
Table 3. Summary of structural MRI studies examining cognitive status subgroups.

\begin{tabular}{|c|c|c|c|c|c|c|c|c|c|c|}
\hline \multirow[t]{3}{*}{ Author (year) } & \multirow[t]{3}{*}{ Sample age $(n)$} & \multirow[t]{3}{*}{ Structural modality } & \multicolumn{8}{|c|}{ Effect of Cognitive Status } \\
\hline & & & \multicolumn{2}{|c|}{ Frontal } & \multicolumn{2}{|c|}{ MTL } & \multicolumn{2}{|c|}{ Posterior } & \multicolumn{2}{|c|}{ Whole brain } \\
\hline & & & $\mathrm{YO}$ & $\mathrm{OO}$ & $\mathrm{YO}$ & $\mathrm{OO}$ & $\mathrm{YO}$ & $\mathrm{OO}$ & YO & $\mathrm{OO}$ \\
\hline \multicolumn{11}{|l|}{ Volumetry \& Morphometry } \\
\hline Stricker et al. (2011) & $60-91(230)$ & Cortical thickness & & & + & o & + & o & & \\
\hline Barkhof et al. (2007) & $85-105(132)$ & Volume & & & & + & & & & \\
\hline Gosche et al. (2002) & $87-93(56)$ & Volume & & & & + & & & & \\
\hline Lopez et al. (2014) & $72-96(183)$ & Volume & & & + & + & & & & \\
\hline Yang et al. (2016b) & $71-103(244)$ & Volume & + & + & + & + & + & + & & \\
\hline Holland et al. (2012) & $65-90(723)$ & Volume & & & + & + & + & o & + & o \\
\hline Rosano et al. (2012) & $\mathrm{M}=83(258)$ & $\Delta$ Volume & & & & - & & & & \\
\hline \multicolumn{11}{|c|}{ White matter hyperintensities \& Microstructure } \\
\hline Rosano et al. (2012) & $\mathrm{M}=83(258)$ & $\Delta \mathrm{MD}$ & & - & & & & - & & \\
\hline Zamboni et al. (2019) & $20-102(566)$ & WMH, FA, MD & + & o & & & & & + & o \\
\hline Polvikoski et al. (2010) & $85-104(132)$ & $\mathrm{WMH}$ & & + & & & & & & \\
\hline Tanskanen et al. (2013) & $85-105(123)$ & WMH & & & & & & & & o \\
\hline Yang et al. (2016b) & $71-103(244)$ & WMH & & & & & & & o & o \\
\hline Rosano et al. (2012) & $\mathrm{M}=83(258)$ & $\Delta \mathrm{WMH}$ & & & & & & & & o \\
\hline
\end{tabular}

Notes. For each structural modality and brain region, symbols indicate observations of better (+), worse (-), or no difference (o) in brain structure (i.e., smaller volume, more white matter hyperintensities [WMH], higher diffusivity for cognitively normal youngerold (YO) and/or oldest-old (OO) age groups relative to those with cognitive impairment or Alzheimer's disease and other dementias. Volumetry and morphometry studies are sorted by age effects in the medial temporal lobe (MTL). WMH and microstructure studies are sorted by age effects in the frontal lobe followed by the whole brain. $\mathrm{FA}=$ fractional anisotropy, $\mathrm{MD}=$ mean diffusivity, $\Delta=$ longitudinal change. 
Table 4. Summary of functional MRI studies.

\begin{tabular}{|c|c|c|c|c|c|}
\hline \multirow[t]{2}{*}{ Author (year) } & \multirow[t]{2}{*}{ Sample age $(n)$} & \multirow[t]{2}{*}{ Cognitive domain } & \multicolumn{3}{|c|}{ Effect of Age on BOLD Activity } \\
\hline & & & Frontal & MTL & Posterior \\
\hline \multicolumn{6}{|l|}{ Task-related fMRI } \\
\hline Beeri et al. (2011) & $70-90+(29)$ & Recognition memory & $\mathrm{O}$ & - & - \\
\hline Wang et al. (2009) & $64-96(34)$ & Recognition memory & o & o & - \\
\hline Rosano et al. (2005a) & $12-82+(28)$ & Executive control & - & & - \\
\hline Rosano et al. (2005b) & $\mathrm{M}=80-82(16)$ & Executive control & $+\mathrm{CI}>\mathrm{CN}$ & & $+\mathrm{Cl}>\mathrm{CN}$ \\
\hline Kennedy et al. (2015) & $20-89(316)$ & Semantic judgments & - & - & - \\
\hline Resting state $f M R I$ & & & Default mode & Frontoparietal & Motor \\
\hline Prawiroharjo et al. (2020) & $65-80+(44)$ & Recall memory & + & & + \\
\hline Jiang et al. (2020) & $76-103(104)$ & Visuospatial task & - & + & + \\
\hline Li et al. (2020) & $M=82(34)$ & $\Delta$ General cognition & + & & + \\
\hline
\end{tabular}

Notes. For each functional modality and brain region (task-related) or network (resting state), symbols indicate positive $(+)$, negative (-), or non-significant (o) associations between age and BOLD activity. All studies are sorted by the cognitive domain examined. Superscripts indicate that the effect was seen for cognitively impaired (CI) versus normal (CN) subgroups. $\Delta=$ longitudinal change. 